\title{
Domain Walls and M2-Branes Partition Functions: M-Theory and ABJM Theory
}

\author{
M. Nouman Muteeb \\ Abdus Salam School of Mathematical Sciences, Lahore, Pakistan
}

\begin{abstract}
We study the BPS counting functions (free energies) of the M-string configurations. We consider separated M5-branes along with M2-branes stretched between them, with M5-branes acting as domain walls interpolating different configurations of M2-branes. We find recursive structure in the free energies of these configurations. The M-string degrees of freedom on the domain walls are interpreted in terms of a pair of interacting supersymmetric WZW models. We also compute the elliptic genus of the M-string in a toy model of the ABJM theory and compare it with the M-theory computation.
\end{abstract}

Keywords: open topological string wave function, gauged WZW models, domain walls

DOI: 10.31526/LHEP.2021.202

\section{DOMAIN WALLS IN M-THEORY: AN INTRODUCTION}

The study of classification and dynamics of the $6 \mathrm{~d}$ Supersymmetric CFTs (SCFTs) is one of the important problems that is currently an active area of research. The maximally supersymmetric $6 \mathrm{~d}$ CFTs are called $(2,0)$ theories. The type IIB string theory in the background of A-type gives rise to $(2,0)$ A-type theory. In the M-theory formulation, the $(2,0) A_{N-1}$ theory is the worldvolume theory of $N$ parallel and coincident M5-branes. Away from the conformal point, the CFT describes the dynamics of the self-dual strings of small tensions. In the M-theory, these strings are described by the one-dimensional intersections of M5-branes and M2-branes. The strings support $(4,0)$ quiver gauge theory. One crucial result of $[1,2,3,4,5]$ is that the elliptic genus of this quiver gauge theory turns out to be equal to the partition function of the bulk theory.

The superconformal group of the theory is $\operatorname{Osp}(2,6 \mid 4)$. Let's denote the $11 \mathrm{~d}$ space-time $\mathbb{R}^{1,10}$ by the coordinates $x^{i}, i=0,1, \ldots, 10$. The coincident M5-branes span the coordinates $\left\{x^{0}, x^{1}, \ldots, x^{5}\right\}$. In the nonconformal limit, the M5-branes are separated along the $x^{6}$ direction with positions denoted by $a_{i}, i=1,2, \ldots, N$. The M2-branes are suspended between consecutive M5-branes and span the coordinates $\left\{x^{0}, x^{1}, x^{6}\right\}$. The M2-brane couples to a 2-from field $B$ inside the M5-brane worldvolume, and the boundary of the M2-brane is what is called the M-string. If we denote by $\Gamma^{I}$ the $32 \times 3211 \mathrm{~d}$ gamma matrices, then the supersymmetries preserved by the M-string are given by

$$
\Gamma^{016} \epsilon=\epsilon, \quad \Gamma^{012345} \epsilon=\epsilon, \quad \Gamma^{01} \epsilon=\epsilon,
$$

where $\epsilon$ is the 32-component spinor and $\Gamma^{I_{1} I_{2} \ldots I_{k}}=\Gamma^{I_{1}} \Gamma^{I_{2}} \ldots \Gamma^{I_{k}}$.

It is interesting to compact $x^{1}$ to a circle of radius $R_{1}$ and consider M-theory compactification on this circle. This gives rise to $N=2^{*} \mathrm{SYM}$ in the transverse five dimensions. The gauge coupling constant is given by $g_{Y M}^{2}=4 \pi^{2} R_{1}$. The momentum around the $S^{1}$ defines a quantum number of the $5 \mathrm{~d}$ BPS particles $\frac{k}{R_{1}}=-\frac{1}{8 g_{Y M}^{2}} \int d^{4} x \operatorname{tr}(F \wedge F)$ and with the corresponding mass $M=R_{1} \delta_{i j}+\frac{k}{R_{1}}$, where $\delta_{i j}$ denotes the separation between $i$-th and $j$-th M5-branes. Moreover, the mass deformation also breaks the string worldsheet supersymmetry $(4,4)$ to $(4,0)$.

A further compactification of the theory can be considered along the compactified direction $x^{0}$. This makes the worldvolume of the M5-branes to be $\mathbb{R}^{4} \times \mathrm{T}^{2}$. Twisting the theory as one moves around the second $S^{1}$ defines the so-called $\Omega$ background which makes it possible to apply the equivariant localisation to compute the partition function. To engineer $5 \mathrm{~d}$ $\mathcal{N}=1^{*} S U(N)$ gauge theory, one has to compact M-theory on the elliptic CY3-fold $A_{N-1} \times \mathrm{T}^{2}$. The instantons in the $4 \mathrm{~d}$ theory are none other than the M-strings wrapped on the whole of $\mathrm{T}^{2}$.

Recall that the symmetry $U(1)_{\epsilon_{1}} \times U(2)_{\epsilon_{2}} \times U(1)_{m}$ acts on the two $\mathbb{R}^{4}$ s defined by the coordinates $\mathbb{R}_{\|}^{4}: z_{1}=$ $x_{2}+i x_{3}, z_{2}=x_{4}+i x_{5}$ and $\mathbb{R}_{\perp}^{4}: z_{3}=x_{7}+i x_{8}, z_{4}=$ $x_{9}+i x_{10}$ as follows: $\left(z_{1}, z_{2}\right) \rightarrow\left(q z_{1}, t^{-1} z_{2}\right),\left(z_{3}, z_{4}\right) \rightarrow$ $\left(\sqrt{\frac{t}{q}} e^{\pi i(2 m)} z_{3}, \sqrt{\frac{t}{q}} e^{\pi i(-2 m)} z_{4}\right)$ where $q:=e^{2 \pi i \epsilon_{1}}, t:=e^{-2 \pi i \epsilon_{2}}$. The toric geometry, dual to the type IIB D5-NS5-(1,1) branes web, underlying the M-string computation is given in Figure 2. The diagonal edges correspond to the mass parameter $m$, the horizontal direction is periodic with period $\tau$, and $Q$ is the fugacity corresponding to the internal vertical lines.

The target space of the gauged linear sigma model on the worldsheet of M-string is the singular space $\operatorname{Sym}^{n} \mathbb{R}^{4} / S^{n}$ described as the configuration space of $n$ points on $\mathbb{R}^{4}$ modded by the permutation group $S_{n}$. The singularity is due to the coincidence of multiple points, and resolving this singularity gives rise to the Hilbert scheme of $n$ points on $\mathbb{C}^{2}$. So instead of dealing with the ill-defined sigma model on $\operatorname{Sym}^{n} \mathbb{R}^{4} / S^{n}$, one can work with a $(4,0)$ sigma model on the $\mathcal{M}=\operatorname{Hilb}^{n}\left(\mathbb{C}^{2}\right)[1]$. The matter content of the theory is given by the sections of different bundles on the instanton moduli space. In the current situation, we have tangent bundle $T_{\mathcal{M}}$ and the complex tautological bundle $E$. The bundle $E$ corresponds to the contribution of fundamental hypermultiplet in the gauge theory instanton computation. In this theory, the left-handed fermions are the sections of tangent bundle $T_{\mathcal{M}}$, whereas the right-handed fermions are the sections of $E \oplus E^{*}$. The combination $E \oplus E^{*}$, corresponding to the two hypermultiplets, signifies the fact that in the toric geometry $\mathbb{P}^{1}$ of the second hypermultplet is flopped [1].

The M-string worldsheet theory defines the target space $\operatorname{Hilb}^{n}\left(\mathbb{C}^{2}\right)$.

The coupling of the left moving fermions and right moving fermions to different bundles correspond to the different boundary conditions around the 1 -cycles of $\mathrm{T}^{2}$ when comput- 


\begin{tabular}{|l|ccccccccccc|}
\hline \multicolumn{110}{|c|}{ 11d M-theory space-time } \\
\hline & $x^{0}$ & $x^{1}$ & $x^{2}$ & $x^{3}$ & $x^{4}$ & $x^{5}$ & $x^{6}$ & $x^{7}$ & $x^{8}$ & $x^{9}$ & $x^{10}$ \\
\hline M5 & $\times$ & $\times$ & $\times$ & $\times$ & $\times$ & $\times$ & & & & & \\
M2 & $\times$ & $\times$ & & & & & $\times$ & & & & \\
M-string & $\times$ & $\times$ & & & & & & & & & \\
$\epsilon_{1}$ & & $\times$ & $\times$ & & & $\times$ & $\times$ & $\times$ & $\times$ & & \\
$\epsilon_{2}$ & & & $\times$ & $\times$ & & $\times$ & $\times$ & $\times$ & $\times$ & & \\
$m$ & & & & & $\times$ & $\times$ & $\times$ & $\times$ & & & \\
\hline
\end{tabular}

FIGURE 1: M-theory vacuum.

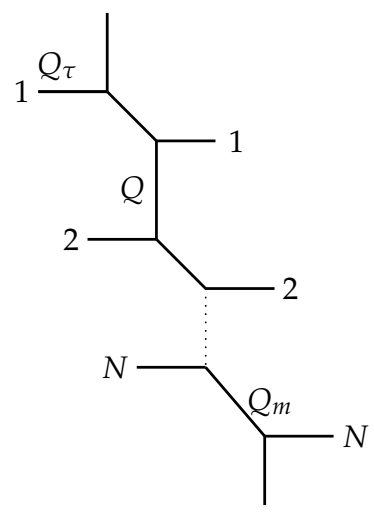

FIGURE 2: Horizontally compactified toric web with corresponding fugacities $Q_{\tau}=e^{2 \pi i \tau}, Q_{m}=e^{2 \pi i m}$, and $Q$.

ing the elliptic genus. The $(2,2)$ sigma model contains bosons $\phi^{i}$ and the fermions $\psi_{-}^{i}, \psi_{-}^{\bar{i}}, \psi_{+}^{i} \psi_{+}^{\bar{i}}$. Locally, the bosons describe a map $\Phi$ : world - sheet $\rightarrow \mathcal{M}$. If we denote by $K$ the bundle of $(1,0)$-forms and by $\bar{K}$ the bundle of $(0,1)$ forms, then the fermions are defined by the following pullback maps:

$$
\begin{array}{ll}
\psi_{-}^{i}=K^{\frac{1}{2}} \otimes \Phi^{*} T_{\mathcal{M}}, & \psi_{-}^{\bar{i}}=K^{\frac{1}{2}} \otimes \Phi^{*} \bar{T}_{\mathcal{M}}, \\
\psi_{+}^{i}=\bar{K}^{\frac{1}{2}} \otimes \Phi^{*} T_{\mathcal{M}}, & \psi_{+}^{\bar{i}}=\bar{K}^{\frac{1}{2}} \otimes \Phi^{*} \bar{T}_{\mathcal{M}} .
\end{array}
$$

If right moving fermions $\eta_{a}$ are also included, the supersymmetry gets broken to $(2,0)$, and we define

$$
\eta^{a}=\bar{K}^{\frac{1}{2}} \otimes \Phi^{*}\left(E \oplus E^{*}\right)
$$

In mathematical terms, the elliptic genus is defined by the index of the following formal combination of vector bundles $V_{Q_{\tau}, y}[1,4]$ :

$$
\begin{aligned}
V_{Q_{\tau}, Q_{m}}= & \bigotimes_{k=0}^{\infty} \wedge_{-Q_{m} Q_{\tau}^{k-1}}\left(E \oplus E^{*}\right) \otimes \bigotimes_{k=1}^{\infty} \wedge_{-Q_{m}^{-1} Q_{\tau}^{k}}\left(E \oplus E^{*}\right)^{*} \\
& \otimes \bigotimes_{k=1}^{\infty} S_{Q_{\tau}^{k}} T_{\mathcal{M}}^{*} \otimes \bigotimes_{k=1}^{\infty} S_{Q_{\tau}^{k}} T_{\mathcal{M}},
\end{aligned}
$$

where $\wedge_{x} W=\sum_{k \geq 0}\left(\wedge^{k} W\right) x^{k}$ and $S_{x} W=\sum_{k \geq 0}\left(S^{k} W\right) x^{k}$ are formal power series. They define the exterior powers and the symmetric powers of a bundle $W$ as coefficients. The partition function $\mathrm{Z}$ of the $\mathrm{M}$-string configuration is the generating function of the elliptic genus $\chi\left(M, V_{Q_{\tau}, y}\right)$ :

$$
\begin{aligned}
Z & =\sum_{k \geq 0} Q^{k} \chi\left(M, V_{Q_{\tau}, y}, k\right) \\
& =\sum_{k \geq 0} Q^{k} \int_{\mathcal{M}} \operatorname{ch}\left(\left(E \oplus E^{*}\right)_{Q_{\tau}, y}\right) \operatorname{Td}\left(T_{\mathcal{M}}\right) \\
& =\sum_{k \geq 0}\left(Q_{m} Q\right)^{k} \int_{\mathcal{M}} e^{\sum_{i=1}^{d} \frac{1}{2}\left(x_{i}-\tilde{x}_{i}\right)} \prod_{i=1}^{d} \frac{\theta_{1}\left(\tau ;-m+\frac{\tilde{x}_{i}}{2 \pi i}\right)}{\theta_{1}\left(\tau ; \frac{x_{i}}{2 \pi i}\right)},
\end{aligned}
$$

where $\mathcal{M}=\operatorname{Hilb}^{n}\left[\mathbb{C}^{2}\right], \tilde{x}_{i}$ and $x_{i}$ denote the formal roots of the Chern polynomial of the bundles $E \oplus E^{*}$ and $T \mathcal{M}$, respectively, $\operatorname{ch}(V)$ is the Chern character of the bundle $V, \operatorname{Td}(T)$ is the Todd class of the tangent bundle, and in the second equality of the last expression, we have used the identity

$$
\begin{aligned}
\operatorname{ch} & \left(\mathrm{V}_{Q_{\tau}, Q_{m}}\right) \operatorname{Td}\left(\mathrm{T}_{\mathcal{M}}\right) \\
& =\prod_{k=1}^{\infty} \frac{\prod_{i=1^{r}}\left(1-Q_{\tau}^{k-1} Q_{m} e^{-\tilde{x}_{i}}\right)\left(1-Q_{\tau}^{k} Q_{m}^{-1} e^{\tilde{x}_{i}}\right)}{\prod_{j=1}^{d} x_{j}^{-1}\left(1-Q_{\tau}^{k-1} e^{-x_{j}}\right)\left(1-Q_{\tau}^{k} e^{x_{j}}\right)}
\end{aligned}
$$

along with the following definition of Dedekind theta function $\theta_{1}(\tau)$ :

$$
\begin{aligned}
\theta_{1}(\tau ; z)= & -i e^{\frac{i \pi \tau}{4}} e^{i \pi z} \prod_{k=1}^{\infty}\left(1-e^{2 \pi i k \tau}\right)\left(1-e^{2 \pi i k \tau} e^{2 \pi i z}\right) \\
& \times\left(1-e^{2 \pi i(k-1) \tau} e^{-2 \pi i z}\right), \\
\theta_{1}(\tau+1 ; z)= & \theta_{1}(\tau ; z), \quad \theta_{1}\left(-\frac{1}{\tau} ; \frac{z}{\tau}\right)=-i(i \tau)^{\frac{1}{2}} e^{\frac{i \pi z^{2}}{\tau}} \theta_{1}(\tau ; z) .
\end{aligned}
$$

Recall that $V=E \oplus E^{*}$ is a bundle on $\mathrm{Hilb}^{k}\left[\mathbb{C}^{2}\right]$. If an ideal I denotes a point of $\mathrm{Hilb}^{k}\left[\mathbb{C}^{2}\right]$, then the fiber of the bundle $V$ over I was found to be [1]

$$
\left.V\right|_{I}=\operatorname{Ext}^{1}(\mathcal{O}, \mathrm{I}) \otimes L^{-\frac{1}{2}} \oplus \operatorname{Ext}^{1}(\mathrm{I}, \mathcal{O}) \otimes L^{-\frac{1}{2}},
$$

where $L$ is the canonical line bundle on $C^{2}$. It has been shown that the appearance of the Ext-groups is related [6] to the counting of open string states between the D-branes wrapped on the holomorphic submanifolds. By determining the equivariant weights of the bundle $V$, the M-string partition function is determined. Intuitively, each M5-brane with M2-branes ending on the left and right gives rise to a factor $\operatorname{Ext}^{1}(\mathrm{I}, \mathrm{J}) \otimes L^{-\frac{1}{2}}$ in the bundle, where I denotes a point of $\mathrm{Hilb}^{n}\left[\mathbb{C}^{2}\right]$ corresponding to the M2-brane on the left and J denotes a point of $\mathrm{Hilb}^{m}\left[\mathrm{C}^{2}\right]$ corresponding to the M2-brane on the right.

For general moduli space $\mathcal{M}_{k_{1}, \ldots, k_{N-1}}=\operatorname{Hilb}^{k_{1}}\left[\mathrm{C}^{2}\right] \times$ Hilb $^{k_{2}}\left[\mathbb{C}^{2}\right] \times \ldots \times$ Hilb $^{k_{N-1}}\left[\mathbb{C}^{2}\right]$, the fiber of the corresponding bundle $V$ over $\left(\mathrm{I}_{1}, \ldots, \mathrm{I}_{N-1}\right) \in \mathcal{M}_{k_{1}, \ldots, k_{N-1}}$ is given by

$$
\left.V\right|_{\left(\mathrm{I}_{1}, \ldots, \mathrm{I}_{N-1}\right)}=\left(\oplus_{a=0}^{N-1} \operatorname{Ext}^{1}\left(\mathrm{I}_{a}, \mathrm{I}_{a+1}\right) \otimes L^{-\frac{1}{2}}\right) .
$$

The fixed points are in one-to-one correspondence with the set of partitions $\left(v_{1}, \ldots, v_{N-1}\right)$, and the equivariant weights of $V$ over the fixed point are

$$
\begin{gathered}
\left\{Q_{m} q^{-i+\frac{1}{2}} t^{j-\frac{1}{2}} \mid(i, j) \in v_{1}\right\} \cup\left\{Q_{m} q^{i-\frac{1}{2}} t^{-j+\frac{1}{2}} \mid(i, j) \in v_{N-1}\right\} \\
\left(\cup_{a=1}^{N-2}\left\{Q_{m} q^{v_{a, j}^{t}-i+\frac{1}{2}} t^{v_{a+1, i}-j+\frac{1}{2}} \mid(i, j) \in v_{a}\right\}\right. \\
\left.\cup\left\{Q_{m} q^{-v_{a+1, j}^{t}+i-\frac{1}{2}} t^{-v_{a, i}+j-\frac{1}{2}} \mid(i, j) \in v_{a+1}\right\}\right) .
\end{gathered}
$$

Using these weights at the fixed points, the partition function turns out to be 


$$
\begin{aligned}
Z_{N} & \left(\tau, m, t_{f_{1}}, t_{f_{2}}, \ldots, t_{f_{N}}, \epsilon_{1}, \epsilon_{2}\right) \\
= & \sum_{k_{1}, k_{2}, \ldots, k_{N-1}}\left(\prod_{a=1}^{N-1}\left(-Q_{f_{a}}\right)^{\left|v_{a}\right|}\right) \sum_{\left|v_{1}\right|=k_{1}, \ldots,\left|v_{N-1}\right|=k_{N-1}} \\
& \times \prod_{a=1}^{N-1} \prod_{(i, j) \in v_{a}} \frac{\theta_{1}\left(\tau ; z_{i, j}^{a}\right) \theta_{1}\left(\tau ; v_{i, j}^{a}\right)}{\theta_{1}\left(\tau ; w_{i, j}^{a}\right) \theta_{1}\left(\tau ; u_{i, j}^{a}\right)}
\end{aligned}
$$

where

$$
\begin{aligned}
z_{i, j}^{a} & =-m+\epsilon_{1}\left(-v_{a-1, j}^{t}+i-\frac{1}{2}\right)-\epsilon_{2}\left(-v_{a, i}+j-\frac{1}{2}\right), \\
v_{i, j}^{a} & =-m+\epsilon_{1}\left(v_{a+1, j}^{t}-i+\frac{1}{2}\right)-\epsilon_{2}\left(v_{a, i}-j+\frac{1}{2}\right), \\
w_{i, j}^{a} & =\epsilon_{1}\left(v_{a, j}^{t}-i\right)-\epsilon_{2}\left(v_{a, i}-j+1\right), \\
u_{i, j}^{a} & =\epsilon_{1}\left(-v_{a, j}^{t}+i-1\right)-\epsilon_{2}\left(-v_{a, i}+j\right), \\
v_{0} & =0, \quad v_{N}=0,
\end{aligned}
$$

and $Q_{f_{a}}:=Q_{a}=e^{2 \pi i t_{f a}}, a=1, \ldots, N-1$, are the fugacities in terms of the coulomb branch parameters $t_{f_{a}}$ that determine the distance between consecutive M5-branes. From the expression (11), we can isolate the following part:

$$
Z_{k_{1}, k_{2}, \ldots, k_{N-1}}=\sum_{\left|v_{1}\right|=k_{1}, \ldots,\left|v_{N-1}\right|=k_{N-1}} \prod_{a=1}^{N-1} \prod_{(i, j) \in v_{a}} \frac{\theta_{1}\left(\tau ; z_{i, j}^{a}\right) \theta_{1}\left(\tau ; v_{i, j}^{a}\right)}{\theta_{1}\left(\tau ; w_{i, j}^{a}\right) \theta_{1}\left(\tau ; u_{i, j}^{a}\right)}
$$

which can be interpreted [7] as the partition function of the following configuration of the wrapped M2-branes: $k_{1}$ M2-branes between the 1st and 2nd M5-branes, $k_{2}$ M2-branes between the 2nd and 3rd M5-branes, and so on up to $k_{N-1}$ M2-branes between the $(N-2)$-th and $(N-1)$-th M5-branes.

After stripping off the gauge theory $U(1)$ part $N P \log Z_{1}$ from the free energy

$$
\begin{aligned}
\Omega_{N}\left(\tau, m, t_{f_{a}}, \epsilon_{1}, \epsilon_{2}\right) & =\operatorname{PLog} Z_{N}\left(\tau, m, t_{f_{a}}, \epsilon_{1}, \epsilon_{2}\right) \\
& =\operatorname{NPLog} Z_{1}+\operatorname{PLog} \tilde{Z}_{N},
\end{aligned}
$$

one can expand the remaining free energy $\tilde{\Omega}\left(\tau, m, t_{f_{a}}, \epsilon_{1}, \epsilon_{2}\right):=$ PLog $\tilde{Z}_{N}$ in terms of the fugacities $Q_{f_{a}}$ as follows:

$$
\begin{aligned}
\tilde{\Omega}\left(\tau, m, t_{f_{a}}, \epsilon_{1}, \epsilon_{2}\right) & :=\operatorname{PLog} \tilde{Z}_{N} \\
& =\sum_{k_{i}=1} Q_{f_{1}}^{k_{1}} \ldots Q_{f_{N-1}}^{k_{N-1}} F_{k_{1}, k_{2}, \ldots, k_{N-1}}\left(\tau, m, \epsilon_{1}, \epsilon_{2}\right),
\end{aligned}
$$

where the multi-index function $F_{k_{1}, k_{2}, \ldots, k_{N-1}}\left(\tau, m, \epsilon_{1}, \epsilon_{2}\right)$ counts the degeneracies of the M-strings bound states.

\section{Presentation of the Article}

After briefly introducing the M-strings and the corresponding elliptic genus of its worksheet theory in Section 2, we discuss recursive structure in the expressions for free energies corresponding to various configurations of the M2-M5 branes. A general configuration consists of an array of multiple M2branes sandwiched between M5-branes. The M2-brane vacua are labeled by the tuple of integer partitions that correspond to the Young diagrams transforming in different representations. We discuss M2-M5 brane configurations in which the M2branes are labeled by antisymmetric representations and symmetric representations. For these representations, the free energies enjoy a partial recursive structure. For mixed ${ }^{1}$ representa- $^{-}$ tions, the recursive structure is lost except for the configuration shown in Section 3. In Section 3, it is discussed that the open topological string wave function for the configuration M2-M5M2 of branes can be described in terms of two WZW models coupled together. In Section 4, we compute the elliptic genus for the M-strings that arise in the ABJM model. We compare it to the M-string elliptic genus as computed in the M-theory framework.

\section{RECURSIVE STRUCTURE IN THE M-STRING PARTITION FUNCTION}

For the M-string configuration in which a single M2-brane is stretched between consecutive M5-branes, the free energies show interesting recursive structure [4]. For more complicated configurations, the recursive structure is not apparent in the expression for free energies, and the correct objects to decompose are the components $Z_{v_{1} v_{2} \ldots v_{n}}$. In doing the following computations, we will often use the following symmetry of the elliptic genera indices $\left\{\mu_{1}, \ldots, \mu_{n}, \varnothing_{1}, \varnothing_{2}, \ldots, \varnothing_{m}\right\}$ :

$$
Z_{\text {permutation }}\left\{\mu_{1}, \ldots, \mu_{n}, \varnothing_{1}, \varnothing_{2}, \ldots, \varnothing_{m}\right\}=Z_{\mu_{1}, \ldots, \mu_{n}, \varnothing_{1}, \varnothing_{2}, \ldots, \varnothing_{m}},
$$

where $\varnothing_{i}=\varnothing_{j}$ for all $i, j, m$ can be less than, equal to, or greater than $n$ and permutation denotes any possible permutation of the given indices.

For the configuration of partitions $\{\alpha, \alpha, \alpha, \ldots, \alpha\}$,

$$
Z_{\alpha \alpha . . \alpha}=\text { all possible ways of factorizing }+Z_{\alpha} W_{\alpha}^{(k-1)},
$$

where $W_{\alpha}$ can be thought of as a universal factor corresponding to removing a single M5-brane. The meaning of the phrase "all possible ways of factorizing" is the following: we will see explicitly in the next section that there is a recursive structure in the expansion coefficients $Z_{v_{1} v_{2} \ldots v_{n}}$ of the elliptic genera; for example,

$$
Z_{222}-2 Z_{22 \varnothing} Z_{2 \varnothing \varnothing}+Z_{2 \varnothing \varnothing}^{3}=Z_{2 \varnothing \varnothing} W_{2}\left(\tau, m, \epsilon_{1}, \epsilon_{2}\right),
$$

where the explicit expressions for the factors $Z_{222}, Z_{22 \varnothing}, Z_{2 \varnothing \varnothing}$, and $W_{2}\left(\tau, m, \epsilon_{1}, \epsilon_{2}\right)$ are given in the next section. We can also write the last expression as

$$
\begin{aligned}
& Z_{222}=2 Z_{22 \varnothing} Z_{2 \varnothing \varnothing}-Z_{2 \varnothing \varnothing}^{3}+Z_{2 \varnothing \varnothing} W_{2}\left(\tau, m, \epsilon_{1}, \epsilon_{2}\right) \\
& =\text { all possible ways of factorizing }
\end{aligned}
$$

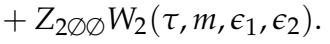

In general, if there are $k$ partitions $\alpha_{1}, \ldots, \alpha_{k}$ of the same size, then it is the case that

$$
\begin{aligned}
Z_{\alpha_{1} \alpha_{2} \ldots \alpha_{k}}= & \text { all possible ways of factorizing } \\
& +Z_{\beta} W_{\alpha_{1} \alpha_{2}} W_{\alpha_{2} \alpha_{3}} \ldots W_{\alpha_{k-2} \alpha_{k-1}} W_{\alpha_{k-1} \alpha_{k}}
\end{aligned}
$$

\footnotetext{
${ }^{1}$ Configurations of M5-M2 branes in which an M2-brane may carry a symmetric or an antisymmetric representation.
} 
where $\beta$ is the result of fusing [8] the partitions $\left(\alpha_{1}, \alpha_{2}, \ldots, \alpha_{k}\right)$. The universal factors $W_{\alpha \beta}$ can be thought of as the effect of removing an M5-brane which fuses partitions $\alpha$ and $\beta$. It should be possible (see Section 3) to obtain $W_{\alpha \beta}$ from the M2-brane perspective as some kind of partition function associated with the domain wall represented by the M5-brane between the vacua labeled by $\alpha$ and $\beta$. This should also be possible to do using the ABJM theory.

The free energy $F=\ln (Z)$ constructed from the partition function contains information about both the single particle and multiparticle BPS states. The plethystic summation is used to project out the multiparticle states. Hence, the function $F_{k_{1} k_{2}, \ldots, k_{n}}$ counts single particle BPS states and can be expanded as

$$
\begin{aligned}
& F_{k_{1}, \ldots, k_{N-1}}\left(\tau, m, \epsilon_{1}, \epsilon_{2}\right) \\
& =\text { coefficient of } Q_{f_{1}}^{k_{1}} \ldots Q_{f_{N-1}}^{k_{N-1}} \\
& \quad \text { in } \sum_{l \geq 1} \frac{\mu(l)}{l} \log \left(Z_{N}\left(l \tau, l m, l t_{f_{a}}, l \epsilon_{1}, l \epsilon_{2}\right)\right) .
\end{aligned}
$$

\subsection{Recursive Structure for the Configuration of Fully Anti- symmetric Young Diagrams}

Below, we give examples for a few configurations of the M5-M2 brane system. These examples show that there is no recursive structure for the full expressions of the free energies. Only a part of the expression of the free energy shows the recursive structure. This part is what is alluded to before as

$$
Z_{\alpha_{1} \alpha_{2} \ldots \alpha_{k}}+\text { all possible ways of factorizing. }
$$

- $F_{2 \varnothing \varnothing,} F_{22 \varnothing,} F_{222}$

$$
\begin{aligned}
& F_{2 \varnothing \varnothing}\left(\tau, m, \epsilon_{1}, \epsilon_{2}\right)=-\frac{1}{2} Z_{1 \varnothing \varnothing}\left(\tau, m, \epsilon_{1}, \epsilon_{2}\right)^{2} \\
& +\left[Z_{2 \varnothing \varnothing}\left(\tau, m, \epsilon_{1}, \epsilon_{2}\right)\right] \\
& -\left(Z_{1 \varnothing \varnothing}\left(2 \tau, 2 m, 2 \epsilon_{1}, 2 \epsilon_{2}\right)\right) \\
& F_{22 \varnothing}\left(\tau, m, \epsilon_{1}, \epsilon_{2}\right)=-\left[Z_{2 \varnothing \varnothing}^{2}\left(\tau, m, \epsilon_{1}, \epsilon_{2}\right)-Z_{22 \varnothing}\left(\tau, m, \epsilon_{1}, \epsilon_{2}\right)\right] \\
& -\left(F^{11 \varnothing}\left(2 \tau, 2 m, 2 \epsilon_{1}, 2 \epsilon_{2}\right)\right)+\text { other terms }
\end{aligned}
$$

and finally,

$$
\begin{aligned}
& F_{222}\left(\tau, m, \epsilon_{1}, \epsilon_{2}\right) \\
& =\left[Z_{2 \varnothing \varnothing}^{3}\left(\tau, m, \epsilon_{1}, \epsilon_{2}\right)-2 Z_{22 \varnothing}\left(\tau, m, \epsilon_{1}, \epsilon_{2}\right) Z_{2 \varnothing \varnothing}\left(\tau, m, \epsilon_{1}, \epsilon_{2}\right)\right. \\
& \left.\quad+Z_{222}\left(\tau, m, \epsilon_{1}, \epsilon_{2}\right)\right]-\left(F_{111}\left(2 \tau, 2 m, 2 \epsilon_{1}, 2 \epsilon_{2}\right)\right) \\
& \quad+\text { other terms. }
\end{aligned}
$$

We now show that the terms in the square brackets form a recursive structure. First, we consider instanton number $k_{i}=2$ and the following Young diagrams:

$$
v_{1}=\{1,1\}, \quad v_{2}=\{1,1\}, \ldots, \text { and so on. }
$$

Using the notation $\theta_{1}(x \pm y):=\theta_{1}(x+y) \theta_{1}(x-y)$, we find

$$
\begin{aligned}
& Z_{22 \varnothing}\left(\tau, m, \epsilon_{1}, \epsilon_{2}\right)-Z_{2 \varnothing \varnothing}^{2}\left(\tau, m, \epsilon_{1}, \epsilon_{2}\right) \\
& \quad=Z_{2 \varnothing \varnothing}\left(\tau, m, \epsilon_{1}, \epsilon_{2}\right) \mathbf{W}_{2}\left(\tau, m, \epsilon_{1}, \epsilon_{2}\right),
\end{aligned}
$$

where

$$
\begin{aligned}
& \mathbf{W}_{2}\left(\tau, m, \epsilon_{1}, \epsilon_{2}\right) \\
& :=\frac{1}{\theta_{1}\left(\epsilon_{1}-\epsilon_{2}\right) \theta_{1}\left(-2 \epsilon_{2}\right) \theta_{1}\left(\epsilon_{1}\right) \theta_{1}\left(-\epsilon_{2}\right)} \\
& \quad \times\left[\theta_{1}\left(-m \pm \frac{\epsilon_{1}}{2} \mp \frac{3 \epsilon_{2}}{2}\right) \theta_{1}\left(-m \pm \epsilon_{-}\right)\right. \\
& \left.\quad \quad-\theta_{1}\left(-m \pm \epsilon_{+}\right) \theta_{1}\left(-m \pm \frac{\epsilon_{1}}{2} \pm \frac{3 \epsilon_{2}}{2}\right)\right]
\end{aligned}
$$

to the next order

$$
\begin{aligned}
Z_{222} & \left(\tau, m, \epsilon_{1}, \epsilon_{2}\right)+Z_{2 \varnothing \varnothing}^{3}\left(\tau, m, \epsilon_{1}, \epsilon_{2}\right) \\
& -2 Z_{22 \varnothing}\left(\tau, m, \epsilon_{1}, \epsilon_{2}\right) Z_{2 \varnothing \varnothing}\left(\tau, m, \epsilon_{1}, \epsilon_{2}\right) \\
= & Z_{2 \varnothing \varnothing}\left(\tau, m, \epsilon_{1}, \epsilon_{2}\right) \mathbf{W}_{2}\left(\tau, m, \epsilon_{1}, \epsilon_{2}\right)^{2} .
\end{aligned}
$$

- $F_{3 \varnothing \varnothing,} F_{33 \varnothing,} F_{333}$

$$
\begin{aligned}
& F_{3 \varnothing \varnothing}\left(\tau, m, \epsilon_{1}, \epsilon_{2}\right)=\left[Z_{\left.3 \varnothing \varnothing\left(\tau, m, \epsilon_{1}, \epsilon_{2}\right)\right]}\right. \\
& -Z_{1 \varnothing \varnothing}\left(\tau, m, \epsilon_{1}, \epsilon_{2}\right) Z_{2 \varnothing \varnothing}\left(\tau, m, \epsilon_{1}, \epsilon_{2}\right) \\
& -\frac{1}{3} Z_{1 \varnothing \varnothing}\left(\tau, m, \epsilon_{1}, \epsilon_{2}\right)^{3} \text {, } \\
& F_{33 \varnothing}\left(\tau, m, \epsilon_{1}, \epsilon_{2}\right)=\left[Z_{33 \varnothing}\left(\tau, m, \epsilon_{1}, \epsilon_{2}\right)-Z_{3 \varnothing \varnothing}\left(\tau, m, \epsilon_{1}, \epsilon_{2}\right)^{2}\right] \\
& + \text { other terms, } \\
& F_{333}\left(\tau, m, \epsilon_{1}, \epsilon_{2}\right)=\left[Z_{333}\left(\tau, m, \epsilon_{1}, \epsilon_{2}\right)-2 Z_{33 \varnothing}\left(\tau, m, \epsilon_{1}, \epsilon_{2}\right)\right.
\end{aligned}
$$

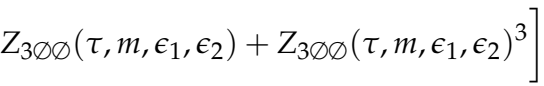

$$
\begin{aligned}
& + \text { other terms. }
\end{aligned}
$$

Now, we consider the terms in square brackets for instanton number $k_{i}=3$ and the following Young diagrams:

$$
\begin{aligned}
& v_{1}=\{1,1,1\}, \quad v_{2}=\{1,1,1\}, \ldots, \text { and so on }
\end{aligned}
$$

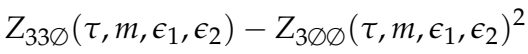

$$
\begin{aligned}
& =Z_{3 \varnothing \varnothing}\left(\tau, m, \epsilon_{1}, \epsilon_{2}\right) \mathbf{W}_{3}\left(\tau, m, \epsilon_{1}, \epsilon_{2}\right),
\end{aligned}
$$

where

$$
\begin{aligned}
\mathbf{W}_{3} & \left(\tau, m, \epsilon_{1}, \epsilon_{2}\right) \\
= & \frac{1}{\theta_{1}\left(\epsilon_{1}-\epsilon_{2}\right) \theta_{1}\left(-2 \epsilon_{2}\right) \theta_{1}\left(\epsilon_{1}\right) \theta_{1}\left(-\epsilon_{2}\right) \theta_{1}\left(-3 \epsilon_{2}\right) \theta_{1}\left(\epsilon_{1}-2 \epsilon_{2}\right)} \\
& \times\left[\theta_{1}\left(-m \pm \frac{\epsilon_{1}}{2} \mp \frac{5 \epsilon_{2}}{2}\right) \theta_{1}\left(-m \pm \frac{\epsilon_{1}}{2} \mp \frac{3 \epsilon_{2}}{2}\right) \theta_{1}\left(-m \pm \epsilon_{-}\right)\right. \\
& \left.-\theta_{1}\left(-m \pm \frac{\epsilon_{1}}{2} \pm \frac{5 \epsilon_{2}}{2}\right) \theta_{1}\left(-m \pm \frac{\epsilon_{1}}{2} \pm \frac{3 \epsilon_{2}}{2}\right) \theta_{1}\left(-m \pm \epsilon_{+}\right)\right]
\end{aligned}
$$

to the next order

$$
\begin{aligned}
& Z_{333}\left(\tau, m, \epsilon_{1}, \epsilon_{2}\right)-2 Z_{33 \varnothing}\left(\tau, m, \epsilon_{1}, \epsilon_{2}\right) Z_{3 \varnothing \varnothing}\left(\tau, m, \epsilon_{1}, \epsilon_{2}\right)
\end{aligned}
$$

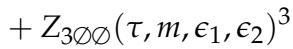

$$
\begin{aligned}
& =Z_{3 \varnothing \varnothing}\left(\tau, m, \epsilon_{1}, \epsilon_{2}\right) \mathbf{W}_{3}\left(\tau, m, \epsilon_{1}, \epsilon_{2}\right)^{2} .
\end{aligned}
$$




\subsection{Observation}

We observe that $W_{i}\left(\tau, m, \epsilon_{1}, \epsilon_{2}\right), i=1, \ldots, N$, follow a pattern

$$
\begin{aligned}
& W_{1}\left(\tau, m, \epsilon_{1}, \epsilon_{2}\right)=\frac{\theta_{1}\left(-m \pm \epsilon_{+}\right)-\theta_{1}\left(-m \pm \epsilon_{-}\right)}{\theta_{1}\left(\epsilon_{1}\right) \theta_{1}\left(-\epsilon_{2}\right)} \\
& W_{2}\left(\tau, m, \epsilon_{1}, \epsilon_{2}\right) \\
& \quad=\frac{\theta_{1}\left(-m \pm \epsilon_{+}\right) \theta_{1}\left(-m \pm \epsilon_{+} \pm \epsilon_{2}\right)-\theta_{1}\left(-m \pm \epsilon_{-}\right) \theta_{1}\left(-m \pm \epsilon_{-} \mp \epsilon_{2}\right)}{\theta_{1}\left(\epsilon_{1}\right) \theta_{1}\left(\epsilon_{1}-\epsilon_{2}\right) \theta_{1}\left(-\epsilon_{2}\right) \theta_{1}\left(-2 \epsilon_{2}\right)}
\end{aligned}
$$$$
W_{3}\left(\tau, m, \epsilon_{1}, \epsilon_{2}\right)
$$$$
\begin{aligned}
= & \frac{\theta_{1}\left(-m \pm \epsilon_{+}\right) \theta_{1}\left(-m \pm \epsilon_{+} \pm \epsilon_{2}\right) \theta_{1}\left(-m \pm \epsilon_{+} \pm 2 \epsilon_{2}\right)}{\theta_{1}\left(\epsilon_{1}\right) \theta_{1}\left(\epsilon_{1}-\epsilon_{2}\right) \theta_{1}\left(\epsilon_{1}-2 \epsilon_{2}\right) \theta_{1}\left(-\epsilon_{2}\right) \theta_{1}\left(-2 \epsilon_{2}\right) \theta_{1}\left(-3 \epsilon_{2}\right)} \\
& -\frac{\theta_{1}\left(-m \pm \epsilon_{-}\right) \theta_{1}\left(-m \pm \epsilon_{-} \mp \epsilon_{2}\right) \theta_{1}\left(-m \pm \epsilon_{-} \mp 2 \epsilon_{2}\right)}{\theta_{1}\left(\epsilon_{1}\right) \theta_{1}\left(\epsilon_{1}-\epsilon_{2}\right) \theta_{1}\left(\epsilon_{1}-2 \epsilon_{2}\right) \theta_{1}\left(-\epsilon_{2}\right) \theta_{1}\left(-2 \epsilon_{2}\right) \theta_{1}\left(-3 \epsilon_{2}\right)} .
\end{aligned}
$$

The above simple observation leads to the following generalization:

$$
\begin{aligned}
W_{N}\left(\tau, m, \epsilon_{1}, \epsilon_{2}\right)= & \prod_{k=1}^{N}\left[\frac{\theta_{1}\left(-m \pm \epsilon_{+} \pm(k-1) \epsilon_{2}\right)}{\theta_{1}\left(-k \epsilon_{2}\right) \theta_{1}\left(\epsilon_{1}-(k-1) \epsilon_{2}\right)}\right] \\
& -\prod_{k=1}^{N}\left[\frac{\theta_{1}\left(-m \pm \epsilon_{-} \mp(k-1) \epsilon_{2}\right)}{\theta_{1}\left(-k \epsilon_{2}\right) \theta_{1}\left(\epsilon_{1}-(k-1) \epsilon_{2}\right)}\right] .
\end{aligned}
$$

It is curious to note that $W_{N}\left(\tau, m, \epsilon_{1}, \epsilon_{2}\right)$ can be written in terms of $W_{1}\left(\tau, m, \epsilon_{1}, \epsilon_{2}\right)$. We can rewrite $W_{1}\left(\tau, m, \epsilon_{1}, \epsilon_{2}\right)$ as

$$
\begin{aligned}
W_{1}\left(\tau, m, \epsilon_{1}, \epsilon_{2}\right)= & \frac{\theta_{1}\left(-m \pm \epsilon_{+}\right)-\theta_{1}\left(-m \pm \epsilon_{-}\right)}{\theta_{1}\left(\epsilon_{1}\right) \theta_{1}\left(-\epsilon_{2}\right)} \\
:= & W_{1}^{+}\left(\tau,-m \pm \epsilon_{+}, \epsilon_{1}, \epsilon_{2}\right) \\
& -W_{1}^{-}\left(\tau,-m \pm \epsilon_{-}, \epsilon_{1}, \epsilon_{2}\right) .
\end{aligned}
$$

Then, $W_{N}\left(\tau, m, \epsilon_{1}, \epsilon_{2}\right)$ can be rewritten in terms of $W_{1}^{+}(\tau,-m \pm$ $\left.\epsilon_{+}, \epsilon_{1}, \epsilon_{2}\right)$-and $W_{1}^{-}\left(\tau,-m \pm \epsilon_{-}, \epsilon_{1}, \epsilon_{2}\right)$-as

$$
\begin{aligned}
& W_{N}\left(\tau, m, \epsilon_{1}, \epsilon_{2}\right) \\
& =\prod_{k=1}^{N}\left[W_{1}^{+}\left(\tau,-m \pm \epsilon_{+} \pm(k-1) \epsilon_{2},-k \epsilon_{2}, \epsilon_{1}-(k-1) \epsilon_{2}\right)\right] \\
& \quad-\prod_{k=1}^{N}\left[W_{1}^{-}\left(\tau,-m \pm \epsilon_{-} \mp(k-1) \epsilon_{2},-k \epsilon_{2}, \epsilon_{1}-(k-1) \epsilon_{2}\right)\right] .
\end{aligned}
$$

Note that under the modular transformation $\left(\tau, m, \epsilon_{1}, \epsilon_{2}\right) \rightarrow$ $\left(-\frac{1}{\tau}, \frac{m}{\tau}, \frac{\epsilon_{1}}{\tau}, \frac{\epsilon_{2}}{\tau}\right), W_{N}\left(\tau, m, \epsilon_{1}, \epsilon_{2}\right)$ transforms as

$$
\begin{aligned}
& W_{N}\left(-\frac{1}{\tau}, \frac{m}{\tau}, \frac{\epsilon_{1}}{\tau}, \frac{\epsilon_{2}}{\tau}\right) \\
& =\prod_{k=1}^{N} e^{\frac{\pi i}{\tau}\left(2 m^{2}+2\left(\epsilon_{+}+(k-1) \epsilon_{2}\right)^{2}-k \epsilon_{2}^{2}\right)}\left[\frac{\theta_{1}\left(-m \pm \epsilon_{+} \pm(k-1) \epsilon_{2}\right)}{\theta_{1}\left(-k \epsilon_{2}\right) \theta_{1}\left(\epsilon_{1}-(k-1) \epsilon_{2}\right)}\right] \\
& -\prod_{k=1}^{N} e^{\frac{\pi i}{\tau}\left(2 m^{2}+2\left(\epsilon_{-}-(k-1) \epsilon_{2}\right)^{2}-k \epsilon_{2}^{2}\right)}\left[\frac{\theta_{1}\left(-m \pm \epsilon_{-} \mp(k-1) \epsilon_{2}\right)}{\theta_{1}\left(-k \epsilon_{2}\right) \theta_{1}\left(\epsilon_{1}-(k-1) \epsilon_{2}\right)}\right] .
\end{aligned}
$$

This shows that it is not a modular covariant. However, in the NS limit $\epsilon_{2} \rightarrow 0$, it becomes modular covariant. For finite $N$, in the NS limit $\epsilon_{1} \rightarrow 0, W_{N}\left(\tau, m, \epsilon_{2}\right)$ reduces to the following expression:

$$
\begin{aligned}
& W_{N}\left(\tau, m, \epsilon_{2}\right)^{N S} \\
& =-\frac{\iota}{\eta(\tau)^{3} \theta_{1}\left(-\epsilon_{2}\right) \prod_{k=2}^{N} \theta_{1}\left(-k \epsilon_{2}\right) \theta_{1}\left(-(k-1) \epsilon_{2}\right)} \\
& \quad \times\left(\sum_{l=1}^{N} \theta_{1}^{\prime}\left(-m+\frac{2 l-1}{2} \epsilon_{2}\right) \theta_{1}\left(-m-\frac{2 l-1}{2} \epsilon_{2}\right)\right. \\
& \quad \times \prod_{k=1, k \neq l}^{N} \theta_{1}\left(-m \pm \frac{2 k-1}{2} \epsilon_{2}\right) \\
& \quad-\sum_{l=1}^{N} \theta_{1}^{\prime}\left(-m-\frac{2 l-1}{2} \epsilon_{2}\right) \theta_{1}\left(-m+\frac{2 l-1}{2} \epsilon_{2}\right) \\
& \left.\quad \times \prod_{k=1, k \neq l}^{N} \theta_{1}\left(-m \pm \frac{2 k-1}{2} \epsilon_{2}\right)\right) .
\end{aligned}
$$

\section{Multiwrapping Contribution}

If $n>1$ denotes the number of wrappings, there are two choices.

\section{(a) $n$ Contains Repeated Prime Factors}

In this case, there will be no contributions to the free energy from multiwrappings.

(b) $n$ Is Equal to the Product of $k$ Distinct Prime Factors

For this case, the generalized expression for $W_{N}\left(\tau, m, \epsilon_{1}, \epsilon_{2}\right)$ is given as

$$
\begin{aligned}
W_{n, N} & \left(\tau, m, \epsilon_{1}, \epsilon_{2}\right)^{\text {multi-wrappings }} \\
\equiv & \prod_{k=1}^{N}\left[\frac{\theta_{1}\left(n \tau,-n m \pm n \epsilon_{+} \pm n(k-1) \epsilon_{2}\right)}{\theta_{1}\left(n \tau,-n k \epsilon_{2}\right) \theta_{1}\left(n \tau, n \epsilon_{1}-n(k-1) \epsilon_{2}\right)}\right] \\
& -\prod_{k=1}^{N}\left[\frac{\theta_{1}\left(n \tau,-n m \pm n \epsilon_{-} \mp n(k-1) \epsilon_{2}\right)}{\theta_{1}\left(n \tau,-n k \epsilon_{2}\right) \theta_{1}\left(n \tau, n \epsilon_{1}-n(k-1) \epsilon_{2}\right)}\right] \\
= & W_{N}\left(n \tau, n m, n \epsilon_{1}, n \epsilon_{2}\right) .
\end{aligned}
$$

This result confirms the fact that the correct objects to decompose for multiple M2-brane configurations are the components $Z_{v_{1} v_{2} \ldots v_{n}}$ and not the free energies or BPS degeneracies $F^{\prime}$ s.

\subsection{Mixed Partitions}

- $F_{12 \varnothing \varnothing \varnothing \varnothing, F_{1212} \varnothing \varnothing, F_{121212}}$

$$
\begin{aligned}
& Z_{1212 \varnothing \varnothing}\left(\tau, m, \epsilon_{1}, \epsilon_{2}\right)-Z_{12 \varnothing \varnothing \varnothing \varnothing}^{2}\left(\tau, m, \epsilon_{1}, \epsilon_{2}\right)
\end{aligned}
$$

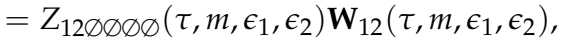


where

$$
\begin{aligned}
& \mathbf{W}_{12}\left(\tau, m, \epsilon_{1}, \epsilon_{2}\right) \\
& =\frac{1}{D_{12 \varnothing \varnothing \varnothing \varnothing\left(\tau, m, \epsilon_{1}, \epsilon_{2}\right)}} \\
& \quad \times \theta_{1}\left(\frac{1}{2}\left(-2 m+\epsilon_{1}-3 \epsilon_{2}\right)\right) \theta_{1}\left(-m-\frac{\epsilon_{1}}{2}-\frac{\epsilon_{2}}{2}\right) \\
& \quad \times \theta_{1}\left(\frac{1}{2}\left(-2 m-\epsilon_{1}+\epsilon_{2}\right)\right) \theta_{1}\left(\frac{1}{2}\left(-2 m+\epsilon_{1}+\epsilon_{2}\right)\right) \\
& \times\left[\theta_{1}\left(\frac{1}{2}\left(-2 m+\epsilon_{1}-\epsilon_{2}\right)\right) \theta_{1}\left(-m-\frac{\epsilon_{1}}{2}+\frac{3 \epsilon_{2}}{2}\right)\right. \\
& \left.\quad \quad-\theta_{1}\left(-m-\frac{\epsilon_{1}}{2}-\frac{\epsilon_{2}}{2}\right) \theta_{1}\left(\frac{1}{2}\left(-2 m+\epsilon_{1}+3 \epsilon_{2}\right)\right)\right] .
\end{aligned}
$$

- $Z_{v_{1} v_{2} v_{3}}$ for $v_{1}=\{1,1,1\}, v_{2}=\{3\}, v_{3}=\{2,1\}$

A nontrivial example of mixed Young diagrams case is $Z_{v_{1} v_{2} v_{3}}$, where $v_{1}$ is the fully symmetric Young diagram, $v_{2}$ is the fully antisymmetric Young diagram, and $v_{3}=\{2,1\}$. Note that $\left|v_{1}\right|=\left|v_{2}\right|=\left|v_{3}\right|=3$. In this case, we get

$$
\begin{aligned}
& Z_{v_{1} v_{2} v_{3}}\left(\tau, m, \epsilon_{1}, \epsilon_{2}\right)-Z_{v_{1}}\left(\tau, m, \epsilon_{1}, \epsilon_{2}\right) Z_{v_{2} v_{3}}\left(\tau, m, \epsilon_{1}, \epsilon_{2}\right) \\
& -Z_{v_{1} v_{2}}\left(\tau, m, \epsilon_{1}, \epsilon_{2}\right) Z_{v_{3}}\left(\tau, m, \epsilon_{1}, \epsilon_{2}\right) \\
& +Z_{v_{1}}\left(\tau, m, \epsilon_{1}, \epsilon_{2}\right) Z_{v_{2}}\left(\tau, m, \epsilon_{1}, \epsilon_{2}\right) Z_{v_{2}}\left(\tau, m, \epsilon_{1}, \epsilon_{2}\right) \\
& =\frac{1}{\theta_{1}\left(-2 \epsilon_{1}\right) \theta_{1}\left(-\epsilon_{1}\right)^{2} \theta_{1}\left(\epsilon_{1}\right)^{2} \theta_{1}\left(3 \epsilon_{1}\right) \theta_{1}\left(\epsilon_{1}-2 \epsilon_{2}\right)^{2} \theta_{1}\left(-\epsilon_{2}+2 \epsilon_{1}\right)^{2} \theta_{1}\left(-3 \epsilon_{2}\right)} \\
& \times \frac{1}{\theta_{1}\left(-\epsilon_{2}\right)^{2} \theta_{1}\left(\epsilon_{2}\right)^{2} \theta_{1}\left(2 \epsilon_{2}\right) \theta_{1}\left(\epsilon_{2}-\epsilon_{1}\right)^{2}} \\
& \times \theta_{1}\left(\frac{1}{2}\left(\epsilon_{1}-3 \epsilon_{2}-2 m\right)\right) \theta_{1}\left(\frac{1}{2}\left(\epsilon_{1}-\epsilon_{2}-2 m\right)\right) \theta_{1}\left(\frac{1}{2}\left(\epsilon_{1}+\epsilon_{2}-2 m\right)\right)^{3} \\
& \theta_{1}\left(\frac{1}{2}\left(3 \epsilon_{1}+\epsilon_{2}-2 m\right)\right) \theta_{1}\left(\frac{1}{2}\left(-\epsilon_{1}+3 \epsilon_{2}-2 m\right)\right) \theta_{1}\left(\frac{1}{2}\left(\epsilon_{1}+3 \epsilon_{2}-2 m\right)\right) \\
& {\left[\theta_{1}\left(\frac{1}{2}\left(5 \epsilon_{1}-3 \epsilon_{2}-2 m\right)\right) \theta_{1}\left(\frac{1}{2}\left(-\epsilon_{1}-\epsilon_{2}-2 m\right)\right) \theta_{1}\left(\frac{1}{2}\left(-\epsilon_{1}+\epsilon_{2}-2 m\right)\right)^{2}\right.} \\
& -\theta_{1}\left(\frac{1}{2}\left(3 \epsilon_{1}+\epsilon_{2}-2 m\right)\right) \theta_{1}\left(\frac{1}{2}\left(5 \epsilon_{1}+\epsilon_{2}-2 m\right)\right) \theta_{1}\left(\frac{1}{2}\left(-\epsilon_{1}+5 \epsilon_{2}-2 m\right)\right) \\
& \left.\theta_{1}\left(\frac{1}{2}\left(\epsilon_{1}+3 \epsilon_{2}-2 m\right)\right)\right] \\
& {\left[\theta_{1}\left(\frac{1}{2}\left(\epsilon_{1}-\epsilon_{2}-2 m\right)\right) \theta_{1}\left(\frac{1}{2}\left(-3 \epsilon_{1}-\epsilon_{2}-2 m\right)\right)^{2} \theta_{1}\left(\frac{1}{2}\left(-\epsilon_{1}-\epsilon_{2}-2 m\right)\right)^{2}\right.} \\
& \theta_{1}\left(\frac{1}{2}\left(-5 \epsilon_{1}+5 \epsilon_{2}-2 m\right)\right) \\
& -\theta_{1}\left(\frac{1}{2}\left(3 \epsilon_{1}-\epsilon_{2}-2 m\right)\right) \theta_{1}\left(\frac{1}{2}\left(5 \epsilon_{1}-\epsilon_{2}-2 m\right)\right) \theta_{1}\left(\frac{1}{2}\left(-3 \epsilon_{1}+\epsilon_{2}-2 m\right)\right) \\
& \theta_{1}\left(\frac{1}{2}\left(5 \epsilon_{1}-\epsilon_{2}-2 m\right)\right) \theta_{1}\left(\frac{1}{2}\left(-3 \epsilon_{1}+\epsilon_{2}-2 m\right)\right) \theta_{1}\left(\frac{1}{2}\left(-\epsilon_{1}+\epsilon_{2}-2 m\right)\right) \\
& \left.\theta_{1}\left(\frac{1}{2}\left(\epsilon_{1}+\epsilon_{2}-2 m\right)\right) \theta_{1}\left(\frac{1}{2}\left(\epsilon_{1}+5 \epsilon_{2}-2 m\right)\right)\right] \text {. }
\end{aligned}
$$

- $Z_{v_{1} v_{2} v_{3}}$ for $v_{1}=\{2,1,1\}, v_{2}=\{3,1\}, v_{3}=\{2,2\}$

A second nontrivial example of mixed Young diagrams corresponds to $v_{1}=\{2,1,1\}, v_{2}=\{3,1\}$, and $v_{3}=\{2,2\}$. Note that $\left|v_{1}\right|=\left|v_{2}\right|=\left|v_{3}\right|=4$ in this case. We get

$$
\begin{aligned}
& Z_{v_{1} v_{2} v_{3}}\left(\tau, m, \epsilon_{1}, \epsilon_{2}\right)-Z_{v_{1}}\left(\tau, m, \epsilon_{1}, \epsilon_{2}\right) Z_{v_{2} v_{3}}\left(\tau, m, \epsilon_{1}, \epsilon_{2}\right) \\
& -Z_{v_{1} v_{2}}\left(\tau, m, \epsilon_{1}, \epsilon_{2}\right) Z_{v_{3}}\left(\tau, m, \epsilon_{1}, \epsilon_{2}\right) \\
& +Z_{v_{1}}\left(\tau, m, \epsilon_{1}, \epsilon_{2}\right) Z_{v_{2}}\left(\tau, m, \epsilon_{1}, \epsilon_{2}\right) Z_{v_{2}}\left(\tau, m, \epsilon_{1}, \epsilon_{2}\right) \\
& =\frac{1}{\theta_{1}\left(-\epsilon_{1}\right)^{2} \theta_{1}\left(\epsilon_{1}\right)^{4} \theta_{1}\left(2 \epsilon_{1}\right) \theta_{1}\left(\epsilon_{1}-3 \epsilon_{2}\right) \theta_{1}\left(\epsilon_{1}-2 \epsilon_{2}\right) \theta_{1}\left(2 \epsilon_{1}-2 \epsilon_{2}\right)^{2} \theta_{1}\left(\epsilon_{1}-\epsilon_{2}\right)^{3}} \\
& \times \frac{1}{\left.\theta_{1}\left(2 \epsilon_{1}-\epsilon_{2}\right) \theta_{1}\left(3 \epsilon_{1}-\epsilon_{2}\right) \theta_{1}\left(-2 \epsilon_{2}\right)^{2} \theta_{1}\left(-\epsilon_{2}\right)^{4}\right) \theta_{1}\left(\epsilon_{2}\right)^{2}} \\
& \theta_{1}\left(\frac{1}{2}\left(\epsilon_{1}-\epsilon_{2}-2 m\right)\right) \theta_{1}\left(\frac{1}{2}\left(\epsilon_{1}+\epsilon_{2}-2 m\right)\right)^{3} \theta_{1}\left(\frac{1}{2}\left(3 \epsilon_{1}+\epsilon_{2}-2 m\right)\right)^{2} \\
& \theta_{1}\left(\frac{1}{2}\left(\epsilon_{1}+3 \epsilon_{2}-2 m\right)\right)^{2} \theta_{1}\left(\frac{1}{2}\left(3 \epsilon_{1}+3 \epsilon_{2}-2 m\right)\right) \\
& {\left[\theta_{1}\left(\frac{1}{2}\left(3 \epsilon_{1}-3 \epsilon_{2}-2 m\right)\right) \theta_{1}\left(\frac{1}{2}\left(\epsilon_{1}-\epsilon_{2}-2 m\right)\right)^{2} \theta_{1}\left(\frac{1}{2}\left(-\epsilon_{1}-\epsilon_{2}-2 m\right)\right)^{2}\right.} \\
& \theta_{1}\left(\frac{1}{2}\left(-3 \epsilon_{1}+\epsilon_{2}-2 m\right)\right)^{2} \theta_{1}\left(\frac{1}{2}\left(-5 \epsilon_{1}+5 \epsilon_{2}-2 m\right)\right) \\
& -\theta_{1}\left(\frac{1}{2}\left(5 \epsilon_{1}-\epsilon_{2}-2 m\right)\right) \theta_{1}\left(\frac{1}{2}\left(-\epsilon_{1}+\epsilon_{2}-2 m\right)\right) \theta_{1}\left(\frac{1}{2}\left(\epsilon_{1}+\epsilon_{2}-2 m\right)\right) \\
& \left.\theta_{1}\left(\frac{1}{2}\left(3 \epsilon_{1}+\epsilon_{2}-2 m\right)\right)^{3} \theta_{1}\left(\frac{1}{2}\left(\epsilon_{1}+3 \epsilon_{2}-2 m\right)\right) \theta_{1}\left(\frac{1}{2}\left(\epsilon_{1}+5 \epsilon_{2}-2 m\right)\right)\right] \\
& {\left[\theta_{1}\left(\frac{1}{2}\left(5 \epsilon_{1}-3 \epsilon_{2}-2 m\right)\right) \theta_{1}\left(\frac{1}{2}\left(\epsilon_{1}-\epsilon_{2}-2 m\right)\right)^{2} \theta_{1}\left(\frac{1}{2}\left(-\epsilon_{1}+\epsilon_{2}-2 m\right)\right)^{2}\right.} \\
& \theta_{1}\left(\frac{1}{2}\left(-3 \epsilon_{1}+3 \epsilon_{2}-2 m\right)\right) \theta_{1}\left(\frac{1}{2}\left(-\epsilon_{1}+3 \epsilon_{2}-2 m\right)\right) \\
& -\theta_{1}\left(\frac{1}{2}\left(3 \epsilon_{1}+\epsilon_{2}-2 m\right)\right) \theta_{1}\left(\frac{1}{2}\left(5 \epsilon_{1}+\epsilon_{2}-2 m\right)\right) \theta_{1}\left(\frac{1}{2}\left(-\epsilon_{1}+5 \epsilon_{2}-2 m\right)\right) \\
& \left.\theta_{1}\left(\frac{1}{2}\left(\epsilon_{1}+3 \epsilon_{2}-2 m\right)\right)^{3} \theta_{1}\left(\frac{1}{2}\left(3 \epsilon_{1}+3 \epsilon_{2}-2 m\right)\right)\right] \text {. }
\end{aligned}
$$

This is in line with the statement given in (20) that if there are $k$ partitions $\alpha_{1}, \ldots, \alpha_{k}$ of the same size, i.e., $\left|\alpha_{1}\right|=\ldots=\left|v_{k}\right|=$ $n$, then it is the case that

$$
\begin{aligned}
Z_{\alpha_{1} \alpha_{2} \ldots \alpha_{k}}= & \text { all possible ways of factorizing } \\
& +Z_{\beta} W_{\alpha_{1} \alpha_{2}} W_{\alpha_{2} \alpha_{3}} \ldots W_{\alpha_{k-2} \alpha_{k-1}} W_{\alpha_{k-1} \alpha_{k}}
\end{aligned}
$$

where $\beta$ is the result of fusing [8] the partitions $\left(\alpha_{1}, \alpha_{2}, \ldots, \alpha_{k}\right)$.

\section{General Configuration}

In the general configuration, we consider $r+1$ M5-branes with $k_{i}, i=1, \ldots, r$, M2-branes between the $i$-th and $(i+1)$-th M5branes. The Young diagrams labeling the M2-branes are in antisymmetric representations. The corresponding components of the elliptic genus $Z_{k_{1} k_{2} \ldots k_{r}}\left(\tau, m, \epsilon_{1}, \epsilon_{2}\right)$ have the following recursive pattern:

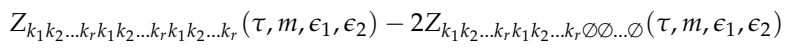

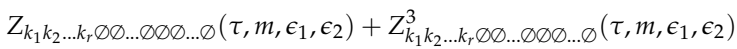

$$
=\left\{\left[\prod_{l=1}^{k_{1}} \theta_{1}\left(-m+\frac{\epsilon_{1}}{2}-\epsilon_{2}\left(k_{2}-l+\frac{1}{2}\right)\right) \theta_{1}\left(-m-\frac{\epsilon_{1}}{2}-\epsilon_{2}\left(l-\frac{1}{2}\right)\right)\right.\right.
$$

$\prod_{l=1}^{k_{2}} \theta_{1}\left(-m+\frac{\epsilon_{1}}{2}-\epsilon_{2}\left(k_{3}-l+\frac{1}{2}\right)\right) \theta_{1}\left(-m-\frac{\epsilon_{1}}{2}-\epsilon_{2}\left(-k_{1}+l-\frac{1}{2}\right)\right)$ 
Letters in High Energy Physics

LHEP-202, 2021

$$
\begin{aligned}
& \left.\prod_{l=1}^{k_{r}} \theta_{1}\left(-m+\frac{\epsilon_{1}}{2}-\epsilon_{2}\left(k_{1}-l+\frac{1}{2}\right)\right) \theta_{1}\left(-m-\frac{\epsilon_{1}}{2}-\epsilon_{2}\left(-k_{r-1}+l-\frac{1}{2}\right)\right)\right] \quad=\left\{\prod_{l=1}^{k_{1}} \theta_{1}\left(-m+\frac{\epsilon_{1}}{2}-\epsilon_{2}\left(k_{2}-l+\frac{1}{2}\right)\right) \theta_{1}\left(-m-\frac{\epsilon_{1}}{2}-\epsilon_{2}\left(l-\frac{1}{2}\right)\right)\right. \\
& \times\left[\prod_{l=1}^{k_{1}} \theta_{1}\left(-m+\frac{\epsilon_{1}}{2}-\epsilon_{2}\left(k_{2}-l+\frac{1}{2}\right)\right) \theta_{1}\left(-m-\frac{\epsilon_{1}}{2}-\epsilon_{2}\left(-k_{r}+l-\frac{1}{2}\right)\right) \quad \prod_{l=1}^{k_{2}} \theta_{1}\left(-m+\frac{\epsilon_{1}}{2}-\epsilon_{2}\left(k_{3}-l+\frac{1}{2}\right)\right) \theta_{1}\left(-m-\frac{\epsilon_{1}}{2}-\epsilon_{2}\left(-k_{1}+l-\frac{1}{2}\right)\right)\right. \\
& \prod_{l=1}^{k_{2}} \theta_{1}\left(-m+\frac{\epsilon_{1}}{2}-\epsilon_{2}\left(k_{3}-l+\frac{1}{2}\right)\right) \theta_{1}\left(-m-\frac{\epsilon_{1}}{2}-\epsilon_{2}\left(-k_{1}+l-\frac{1}{2}\right)\right) \quad \text { : } \\
& \left.\vdots \quad \prod_{l=1}^{k_{r}} \theta_{1}\left(-m+\frac{\epsilon_{1}}{2}-\epsilon_{2}\left(-l+\frac{1}{2}\right)\right) \theta_{1}\left(-m-\frac{\epsilon_{1}}{2}-\epsilon_{2}\left(-k_{r-1}+l-\frac{1}{2}\right)\right)\right\} \\
& \left.\prod_{l=1}^{k_{r}} \theta_{1}\left(-m+\frac{\epsilon_{1}}{2}-\epsilon_{2}\left(k_{1}-l+\frac{1}{2}\right)\right) \theta_{1}\left(-m-\frac{\epsilon_{1}}{2}-\epsilon_{2}\left(-k_{r-1}+l-\frac{1}{2}\right)\right)\right] \quad\left\{\left(\prod_{l=1}^{k_{r}} \theta_{1}\left(-m+\frac{\epsilon_{1}}{2}-\epsilon_{2}\left(k_{1}-l+\frac{1}{2}\right)\right) \theta_{1}\left(-m-\frac{\epsilon_{1}}{2}-\epsilon_{2}\left(-k_{r-1}+l-\frac{1}{2}\right)\right)\right.\right. \\
& \times\left[\prod_{l=1}^{k_{1}} \theta_{1}\left(-m+\frac{\epsilon_{1}}{2}-\epsilon_{2}\left(k_{2}-l+\frac{1}{2}\right)\right) \theta_{1}\left(-m-\frac{\epsilon_{1}}{2}-\epsilon_{2}\left(-k_{r}+l-\frac{1}{2}\right)\right) \quad \times \prod_{l=1}^{k_{1}} \theta_{1}\left(-m+\frac{\epsilon_{1}}{2}-\epsilon_{2}\left(k_{2}-l+\frac{1}{2}\right)\right) \theta_{1}\left(-m-\frac{\epsilon_{1}}{2}-\epsilon_{2}\left(-k_{r}+l-\frac{1}{2}\right)\right)\right. \\
& \prod_{l=1}^{k_{2}} \theta_{1}\left(-m+\frac{\epsilon_{1}}{2}-\epsilon_{2}\left(k_{3}-l+\frac{1}{2}\right)\right) \theta_{1}\left(-m-\frac{\epsilon_{1}}{2}-\epsilon_{2}\left(-k_{1}+l-\frac{1}{2}\right)\right) \quad \prod_{l=1}^{k_{2}} \theta_{1}\left(-m+\frac{\epsilon_{1}}{2}-\epsilon_{2}\left(k_{3}-l+\frac{1}{2}\right)\right) \theta_{1}\left(-m-\frac{\epsilon_{1}}{2}-\epsilon_{2}\left(-k_{1}+l-\frac{1}{2}\right)\right) \\
& \vdots \\
& \left.\left.\prod_{l=1}^{k_{r}} \theta_{1}\left(-m+\frac{\epsilon_{1}}{2}-\epsilon_{2}\left(-l+\frac{1}{2}\right)\right) \theta_{1}\left(-m-\frac{\epsilon_{1}}{2}-\epsilon_{2}\left(-k_{r-1}+l-\frac{1}{2}\right)\right)\right]\right\} \\
& \prod_{l=1}^{k_{r-1}} \theta_{1}\left(-m+\frac{\epsilon_{1}}{2}-\epsilon_{2}\left(k_{r}-l+\frac{1}{2}\right)\right) \\
& -2\left\{\left[\prod_{l=1}^{k_{1}} \theta_{1}\left(-m+\frac{\epsilon_{1}}{2}-\epsilon_{2}\left(k_{2}-l+\frac{1}{2}\right)\right) \theta_{1}\left(-m-\frac{\epsilon_{1}}{2}-\epsilon_{2}\left(l-\frac{1}{2}\right)\right) \quad \theta_{1}\left(-m-\frac{\epsilon_{1}}{2}-\epsilon_{2}\left(-k_{r-2}+l-\frac{1}{2}\right)\right)\right)^{2}\right. \\
& \prod_{l=1}^{k_{2}} \theta_{1}\left(-m+\frac{\epsilon_{1}}{2}-\epsilon_{2}\left(k_{3}-l+\frac{1}{2}\right)\right) \theta_{1}\left(-m-\frac{\epsilon_{1}}{2}-\epsilon_{2}\left(-k_{1}+l-\frac{1}{2}\right)\right) \quad-2\left[\prod_{l=1}^{k_{1}} \theta_{1}\left(-m+\frac{\epsilon_{1}}{2}-\epsilon_{2}\left(k_{2}-l+\frac{1}{2}\right)\right) \theta_{1}\left(-m-\frac{\epsilon_{1}}{2}-\epsilon_{2}\left(l-\frac{1}{2}\right)\right)\right. \\
& \prod_{l=1}^{k_{r}} \theta_{1}\left(-m+\frac{\epsilon_{1}}{2}-\epsilon_{2}\left(k_{1}-l+\frac{1}{2}\right)\right) \theta_{1}\left(-m-\frac{\epsilon_{1}}{2}-\epsilon_{2}\left(-k_{r-1}+l-\frac{1}{2}\right)\right) \\
& \prod_{l=1}^{k_{2}} \theta_{1}\left(-m+\frac{\epsilon_{1}}{2}-\epsilon_{2}\left(k_{3}-l+\frac{1}{2}\right)\right) \theta_{1}\left(-m-\frac{\epsilon_{1}}{2}-\epsilon_{2}\left(-k_{1}+l-\frac{1}{2}\right)\right) \\
& \prod_{l=1}^{k_{1}} \theta_{1}\left(-m+\frac{\epsilon_{1}}{2}-\epsilon_{2}\left(k_{2}-l+\frac{1}{2}\right)\right) \theta_{1}\left(-m-\frac{\epsilon_{1}}{2}-\epsilon_{2}\left(-k_{r}+l-\frac{1}{2}\right)\right) \quad \prod_{l=1}^{k_{r}} \theta_{1}\left(-m+\frac{\epsilon_{1}}{2}-\epsilon_{2}\left(k_{1}-l+\frac{1}{2}\right)\right) \theta_{1}\left(-m-\frac{\epsilon_{1}}{2}-\epsilon_{2}\left(-k_{r-1}+l-\frac{1}{2}\right)\right) \\
& \prod_{l=1}^{k_{2}} \theta_{1}\left(-m+\frac{\epsilon_{1}}{2}-\epsilon_{2}\left(k_{3}-l+\frac{1}{2}\right)\right) \theta_{1}\left(-m-\frac{\epsilon_{1}}{2}-\epsilon_{2}\left(-k_{1}+l-\frac{1}{2}\right)\right) \quad \prod_{l=1}^{k_{1}} \theta_{1}\left(-m+\frac{\epsilon_{1}}{2}-\epsilon_{2}\left(k_{2}-l+\frac{1}{2}\right)\right) \theta_{1}\left(-m-\frac{\epsilon_{1}}{2}-\epsilon_{2}\left(-k_{r}+l-\frac{1}{2}\right)\right)
\end{aligned}
$$

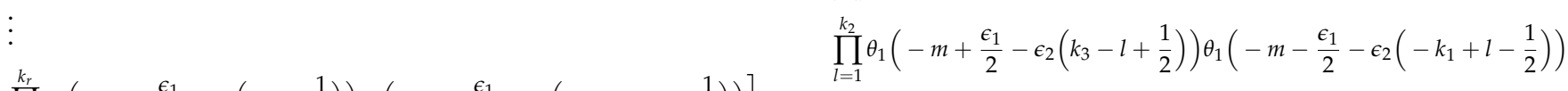

$$
\begin{aligned}
& \left.\prod_{l=1}^{k_{r}} \theta_{1}\left(-m+\frac{\epsilon_{1}}{2}-\epsilon_{2}\left(-l+\frac{1}{2}\right)\right) \theta_{1}\left(-m-\frac{\epsilon_{1}}{2}-\epsilon_{2}\left(-k_{r-1}+l-\frac{1}{2}\right)\right)\right] \quad \text { : } \\
& {\left[\prod_{l=1}^{k_{1}} \theta_{1}\left(-m+\frac{\epsilon_{1}}{2}-\epsilon_{2}\left(k_{2}-l+\frac{1}{2}\right)\right) \theta_{1}\left(-m-\frac{\epsilon_{1}}{2}-\epsilon_{2}\left(l-\frac{1}{2}\right)\right) \quad \prod_{l=1}^{k_{r}} \theta_{1}\left(-m+\frac{\epsilon_{1}}{2}-\epsilon_{2}\left(-l+\frac{1}{2}\right)\right) \theta_{1}\left(-m-\frac{\epsilon_{1}}{2}-\epsilon_{2}\left(-k_{r-1}+l-\frac{1}{2}\right)\right)\right]} \\
& \prod_{l=1}^{k_{2}} \theta_{1}\left(-m+\frac{\epsilon_{1}}{2}-\epsilon_{2}\left(k_{3}-l+\frac{1}{2}\right)\right) \theta_{1}\left(-m-\frac{\epsilon_{1}}{2}-\epsilon_{2}\left(-k_{1}+l-\frac{1}{2}\right)\right) \quad+\left(\prod_{l=1}^{k_{1}} \theta_{1}\left(-m+\frac{\epsilon_{1}}{2}-\epsilon_{2}\left(k_{2}-l+\frac{1}{2}\right)\right) \theta_{1}\left(-m-\frac{\epsilon_{1}}{2}-\epsilon_{2}\left(l-\frac{1}{2}\right)\right)\right. \\
& \begin{array}{l}
\vdots \\
\left.\left.\prod_{l=1}^{k_{r}} \theta_{1}\left(-m+\frac{\epsilon_{1}}{2}-\epsilon_{2}\left(-l+\frac{1}{2}\right)\right) \theta_{1}\left(-m-\frac{\epsilon_{1}}{2}-\epsilon_{2}\left(-k_{r-1}+l-\frac{1}{2}\right)\right)\right]\right\} \quad \prod_{l=1} \quad \vdots
\end{array} \\
& \prod_{l=1}^{k_{2}} \theta_{1}\left(-m+\frac{\epsilon_{1}}{2}-\epsilon_{2}\left(k_{3}-l+\frac{1}{2}\right)\right) \theta_{1}\left(-m-\frac{\epsilon_{1}}{2}-\epsilon_{2}\left(-k_{1}+l-\frac{1}{2}\right)\right) \\
& +\left\{\prod_{l=1}^{k_{1}} \theta_{1}\left(-m+\frac{\epsilon_{1}}{2}-\epsilon_{2}\left(k_{2}-l+\frac{1}{2}\right)\right) \theta_{1}\left(-m-\frac{\epsilon_{1}}{2}-\epsilon_{2}\left(l-\frac{1}{2}\right)\right)\right. \\
& \prod_{l=1}^{k_{2}} \theta_{1}\left(-m+\frac{\epsilon_{1}}{2}-\epsilon_{2}\left(k_{3}-l+\frac{1}{2}\right)\right) \theta_{1}\left(-m-\frac{\epsilon_{1}}{2}-\epsilon_{2}\left(-k_{1}+l-\frac{1}{2}\right)\right) \\
& \left.\left.\prod_{l=1}^{k_{r}} \theta_{1}\left(-m+\frac{\epsilon_{1}}{2}-\epsilon_{2}\left(-l+\frac{1}{2}\right)\right) \theta_{1}\left(-m-\frac{\epsilon_{1}}{2}-\epsilon_{2}\left(-k_{r-1}+l-\frac{1}{2}\right)\right)\right)^{2}\right\}
\end{aligned}
$$

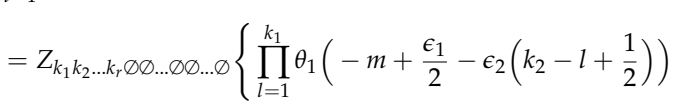

$$
\begin{aligned}
& \theta_{1}\left(-m-\frac{\epsilon_{1}}{2}-\epsilon_{2}\left(l-\frac{1}{2}\right)\right) \\
& \prod_{l=1}^{k_{2}} \theta_{1}\left(-m+\frac{\epsilon_{1}}{2}-\epsilon_{2}\left(k_{3}-l+\frac{1}{2}\right)\right) \theta_{1}\left(-m-\frac{\epsilon_{1}}{2}-\epsilon_{2}\left(-k_{1}+l-\frac{1}{2}\right)\right) \\
& \left.\prod_{l=1}^{k_{r}} \theta_{1}\left(-m+\frac{\epsilon_{1}}{2}-\epsilon_{2}\left(-l+\frac{1}{2}\right)\right) \theta_{1}\left(-m-\frac{\epsilon_{1}}{2}-\epsilon_{2}\left(-k_{r-1}+l-\frac{1}{2}\right)\right)\right\}^{3} \quad \vdots
\end{aligned}
$$

7 


$$
\begin{aligned}
& \prod_{l=1}^{k_{r}} \theta_{1}\left(-m+\frac{\epsilon_{1}}{2}-\epsilon_{2}\left(-l+\frac{1}{2}\right)\right) \theta_{1}\left(-m-\frac{\epsilon_{1}}{2}-\epsilon_{2}\left(-k_{r-1}+l-\frac{1}{2}\right)\right) \\
& -\prod_{l=1}^{k_{1}} \theta_{1}\left(-m+\frac{\epsilon_{1}}{2}-\epsilon_{2}\left(k_{2}-l+\frac{1}{2}\right)\right) \theta_{1}\left(-m-\frac{\epsilon_{1}}{2}-\epsilon_{2}\left(l-\frac{1}{2}\right)\right) \\
& \prod_{l=1}^{k_{2}} \theta_{1}\left(-m+\frac{\epsilon_{1}}{2}-\epsilon_{2}\left(k_{3}-l+\frac{1}{2}\right)\right) \theta_{1}\left(-m-\frac{\epsilon_{1}}{2}-\epsilon_{2}\left(-k_{1}+l-\frac{1}{2}\right)\right) \\
& \left.\prod_{l=1}^{k_{r}} \theta_{1}\left(-m+\frac{\epsilon_{1}}{2}-\epsilon_{2}\left(-l+\frac{1}{2}\right)\right) \theta_{1}\left(-m-\frac{\epsilon_{1}}{2}-\epsilon_{2}\left(-k_{r-1}+l-\frac{1}{2}\right)\right)\right\}^{2}
\end{aligned}
$$

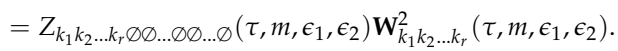

\subsection{Fully Symmetric Configuration of the Young Diagrams}

The symmetric configuration of the Young diagram is the complex conjugate of the antisymmetric configuration, and this amounts to replacing $\epsilon_{1}$ with $\epsilon_{2}$ and vice versa. The results obtained in the previous section remain valid in this situation if we make the replacement

$$
\begin{aligned}
& \epsilon_{2} \leftrightarrow \epsilon_{1} \\
& W_{N}\left(\tau, m, \epsilon_{1}, \epsilon_{2}\right)= \prod_{k=1}^{N}\left[\frac{\theta_{1}\left(-m \pm \epsilon_{+} \pm(k-1) \epsilon_{1}\right)}{\theta_{1}\left(-k \epsilon_{1}\right) \theta_{1}\left(\epsilon_{2}-(k-1) \epsilon_{1}\right)}\right] \\
&-\prod_{k=1}^{N}\left[\frac{\theta_{1}\left(-m \pm \epsilon_{-} \mp(k-1) \epsilon_{1}\right)}{\theta_{1}\left(-k \epsilon_{1}\right) \theta_{1}\left(\epsilon_{2}-(k-1) \epsilon_{1}\right)}\right] .
\end{aligned}
$$

For finite $\mathrm{N}$, in the NS limit $\epsilon_{2} \rightarrow 0$, we get

$$
\begin{aligned}
& W_{N}\left(\tau, m, \epsilon_{2}\right)^{N S} \\
& =-\frac{\iota}{\eta(\tau)^{3} \theta_{1}\left(-\epsilon_{1}\right) \prod_{k=2}^{N} \theta_{1}\left(-k \epsilon_{1}\right) \theta_{1}\left(-(k-1) \epsilon_{1}\right)} \\
& \quad \times\left(\sum_{l=1}^{N} \theta_{1}^{\prime}\left(-m+\frac{2 l-1}{2} \epsilon_{1}\right) \theta_{1}\left(-m-\frac{2 l-1}{2} \epsilon_{1}\right)\right. \\
& \prod_{k=1, k \neq l}^{N} \theta_{1}\left(-m \pm \frac{2 k-1}{2} \epsilon_{1}\right) \\
& -\sum_{l=1}^{N} \theta_{1}^{\prime}\left(-m-\frac{2 l-1}{2} \epsilon_{1}\right) \theta_{1}\left(-m+\frac{2 l-1}{2} \epsilon_{1}\right) \\
& \left.\prod_{k=1, k \neq l}^{N} \theta_{1}\left(-m \pm \frac{2 k-1}{2} \epsilon_{1}\right)\right) .
\end{aligned}
$$

\subsection{Remark: Some Comments on the Algebra of Holomorphic Curves and the Recursive Structure}

Gopakumar and Vafa reformulated $[9,10]$ the topological string amplitudes focussing on the target space perspective. The $5 \mathrm{~d}$ $N=1^{*}$ supersymmetric gauge theory, for a given M-theory CY3-fold compactification, has BPS particles. These BPS particles correspond to the M2-branes wrapped on holomorphic curves in the CY3-fold. The quantum numbers of the BPS particles are given by the curve class $\Sigma \in H_{2}(C Y 3, \mathbb{Z})$ and the spin content of the $5 \mathrm{~d}$ little group of the massive particles $\left(j_{R}, j_{L}\right)=S U(2)_{R} \times S U(2)_{L}$. The particle content with charge $\Sigma$ and spins $\left(j_{R}, j_{L}\right)$ is invariant for a noncompact CY3-fold and is denoted by $N_{\Sigma}^{\left(j_{R}, j_{L}\right)}$. The moduli space furnished by the D2- branes wrapped on $\Sigma$ is topologically nontrivial, and the number of its cohomology classes is equal to $N_{\Sigma}^{\left(j_{R}, j_{L}\right)}$. The explicit form of the topological string partition function is given by

$$
\begin{aligned}
Z(\omega, \epsilon)= & \prod_{\Sigma \in H_{2}(C Y 3)} \prod_{j_{L}} \\
& \times \prod_{k=-j_{L}} \prod_{m=0}^{\infty}\left(1-q^{2 k+m+1} Q^{\Sigma}\right)^{(-1)^{2 j_{L}+1}(m+1) N_{\Sigma}^{\left(j_{R}, j_{L}\right)}},
\end{aligned}
$$

where $Q^{\Sigma}=e^{-T_{\Sigma}}, q=e^{-i \epsilon}$, and $\omega$ is the Kähler form on the CY3-fold. It was shown in [9] that for $j_{L}=0$ the partition function $Z(\omega, \epsilon)$ counts the states in a Hilbert space. It is interesting to note that, for a given particle content $N_{\Sigma}^{\left(j_{R}, j_{L}\right)}$, the above partition function $Z(\omega, \epsilon)$ can be written as an index as [11]

$$
Z=\operatorname{Tr}_{\mathcal{H}}(-1)^{2\left(j_{L}+j_{R}\right)} q^{2 j_{L}^{3}} e^{-T_{\Sigma}},
$$

where $\mathcal{H}$ is the quantized Hilbert subspace containing holomorphic modes of the BPS fields and $T_{\Sigma}$ is the Hamiltonian of the theory. In other words, the topological string partition function can be interpreted as counting the holomorphic (components of the) BPS states in the quantized Hilbert space.

Moreover, as shown in Section 2 in the computation of the M-strings partition function, one has to use fixed point theorems. For that purpose, it is necessary to determine the equivariant weights of certain vector bundles on the M-string moduli space. In the description of the vector bundles, the Ext-groups make an appearance. These groups appear [12] in the counting problem of open string states between the D-branes wrapped on the holomorphic submanifolds.

It was shown in [5] that the free energies $F^{1_{1}, 1_{2}, \ldots, 1_{N}}\left(\tau, m, \epsilon_{1}\right.$, $\epsilon_{2}$ ) for a configuration of finitely separated $N+1$ M5-branes with a single M2-brane stretched between consecutive M5branes are reducible and recursive such that

$$
\begin{aligned}
& F^{1_{1}, 1_{2}, \ldots, 1_{N}}\left(\tau, m, \epsilon_{1}, \epsilon_{2}\right) \\
& \quad=W\left(\tau, m, \epsilon_{1}, \epsilon_{2}\right)^{N-1} F^{1_{1}, 0, \ldots, 0}\left(\tau, m, \epsilon_{1}, \epsilon_{2}\right) .
\end{aligned}
$$

This shows that the factor of $W\left(\tau, m, \epsilon_{1}, \epsilon_{2}\right)$ appears every time a single M5-brane is removed from the configuration -M2-M5-M2-. This recursive structure, as shown in [13], indicates that the degrees of freedom corresponding to the $F^{1_{1}, 1_{2}, \ldots, 1_{N}}\left(\tau, m, \epsilon_{1}, \epsilon_{2}\right)$ can be obtained from $F^{1_{1}, 0, \ldots, 0}\left(\tau, m, \epsilon_{1}, \epsilon_{2}\right)$ up to the universal factor $W\left(\tau, m, \epsilon_{1}, \epsilon_{2}\right)$. A similar interpretation is expected from the generalized $W_{N}\left(\tau, m, \epsilon_{1}, \epsilon_{2}\right)$ computed in Section 2.

The M-string configuration when lifted to the higher dimensional F-theory corresponds [14] to an elliptic CY3-fold in which a D3-brane wraps a $\mathbb{P}^{1}$ whose normal bundle is $\mathcal{O}(-2)$. The configuration of multiple parallel M5-branes with M2-branes stretching between them corresponds to D3-branes wrapping a chain of $\mathbb{P}^{1} \mathrm{~s}$ with $\mathcal{O}(-2)$ normal bundles. The elliptic CY3-fold is a resolved $A_{N-1}$ fibration over the $\mathrm{T}^{2}$. In this setup, the M5-branes correspond to a holomorphic ( -2$)$ curve. In the case under consideration, the holomorphic cycles correspond to the positive roots of the gauge group $S U(N)$ of the $5 d, \mathcal{N}=1^{*}$ gauge theory. Recalling that [15] for two holomorphic curves $C_{1}$ and $C_{2}$, one can compute $\left(C_{1}+C_{2}\right)^{2}=$ $C_{1} \cdot C_{1}+C_{2} \cdot C_{2}+2 C_{1} \cdot C_{2}$ when their self-intersections and $C_{1} \cdot C_{2}$ are known. For the special case of $C_{1} \cdot C_{1}=-2$ and $C_{2} \cdot C_{2}=-2$ 
and $C_{1}$ intersecting $C_{2}$ at a single point, i.e. $C_{1} \cdot C_{2}=1$, we get $\left(C_{1}+C_{2}\right)^{2}=-2-2+2(1)=-2$. Generalizing this to a chain of $(-2)$ curves $C_{1}, C_{2}, \ldots, C_{N}$ in which $i$-th curve intersects only $(i-1)$-th and $(i+1)$-th curves, i.e. $C_{i} \cdot C_{i}=-2, i=1, \ldots, N$, and $C_{i} \cdot C_{i-1}=1, C_{i} \cdot C_{i+1}=1$ with all other intersections equal to zero, it is easy to see that

$$
\left(C_{1}+C_{2}+\ldots+C_{N}\right)^{2}=-2 .
$$

It is interesting to speculate that this property of the $(-2)$ curves mimics the result (53) with the universal factor $W\left(\tau, m, \epsilon_{1}, \epsilon_{2}\right)$ playing the role of the identity element.

As shown in the previous sections, for multiple M2-branes between consecutive M5-branes, instead of the free energies, it is the elliptic genera that carry the recursive structure. It will be interesting to elaborate on this phenomenon in the framework of F-theory.

\section{DOMAIN WALL DEGREES OF FREEDOM: COUPLED SUPERSYMMET- RIC WZW MODELS}

We begin this section by reviewing the supergroup WZW models. The supergroup WZW model $[16,17]$ is described by the maps $f: \Sigma \rightarrow$ (super group)SG from a two-dimensional Euclidean Riemann surface $\Sigma$ to the supergroup $S G$, and its dynamics is given by the action

$$
\begin{aligned}
S[f]= & -\frac{k}{8 \pi} \int_{\Sigma} d^{2} x\left(f^{-1} \partial^{\alpha} f, f^{-1} \partial_{\alpha} f\right) \\
& -\frac{i k}{24 \pi} d^{3} x \epsilon^{\mu \nu \lambda}\left(f^{-1} \partial_{\mu} f,\left[f^{-1} \partial_{\nu} \lambda, f^{-1} \partial_{\lambda} f\right]\right),
\end{aligned}
$$

where $\mathrm{M}$ is a three-manifold with $\Sigma$ as its boundary and $k \in \mathbb{Z}$ is the level. The symmetry group $S G(z) \times S G(\bar{z})$ that generates the left and right actions is defined by

$$
f(z, \bar{z}) \rightarrow \Lambda(z) f(z, \bar{z}) \bar{\Lambda}^{-1}(\bar{z}),
$$

where $\Lambda(z)$ and $\Lambda(\bar{z})$ denote the arbitrary SG-valued functions of the complex variables $z$ and $\bar{z}$. Note that equation (55) is invariant under the transformation given by (56). The conserved currents for this symmetry are given by

$$
J(z)=J^{a}(z) T_{a}=-k \partial_{z} f \cdot f^{-1}
$$

with the generators of the Lie superalgebra sg denoted by $T^{a}$. The OPE of the generators $J^{a}$ is given by

$$
J^{a}(z) J^{b}(w) \sim \frac{k\left(T^{a}, T^{b}\right)}{(z-w)^{2}}+\frac{\left[T^{a}, T^{b}\right]_{c} J^{c}(w)}{z-w} .
$$

As a consequence of the OPE, we have the following commutation relations which define the affine Lie superalgebra sig [17]:

$$
\left[J_{n}^{a}, J_{m}^{n}\right]=\left[T^{a}, T^{b}\right]_{c} J_{n+m}^{c}+m\left(T^{a}, T^{b}\right) \delta_{n+m} k .
$$

On the boundary of an M2-brane, the description by the ABJM model gives rise to the WZW model. The content of the ABJM model can be described in terms of type IIB brane configurations. To this end, see Figure 3, note that the T-duality operation on $\mathrm{D} 3$-branes wrapped on a circle gives rise to D2-branes in

\begin{tabular}{|l|cccccccccc|}
\hline \multicolumn{110}{|c|}{ type IIB space-time } \\
\hline & $x^{0}$ & $x^{1}$ & $x^{2}$ & $x^{3}$ & $x^{4}$ & $x^{5}$ & $x^{6}$ & $x^{7}$ & $x^{8}$ & $x^{9}$ \\
\hline NS5 & $\times$ & $\times$ & $\times$ & $\times$ & $\times$ & $\times$ & & & & \\
D5 & $\times$ & $\times$ & $\times$ & $\times$ & $\times$ & & & $\times$ & & \\
D3 & $\times$ & $\times$ & $\times$ & & & + & & & & \\
D3_ & $\times$ & $\times$ & $\times$ & & & - & & & & \\
\hline
\end{tabular}

FIGURE 3: Type IIB picture

\begin{tabular}{|l|ccccccccccc|}
\hline \multicolumn{110}{|c|}{ 11d M-theory space-time } \\
\hline & $x^{0}$ & $x^{1}$ & $x^{2}$ & $x^{3}$ & $x^{4}$ & $x^{5}$ & $x^{6}$ & $x^{7}$ & $x^{8}$ & $x^{9}$ & $x^{10}$ \\
\hline M5 & $\times$ & $\times$ & & $\times$ & $\times$ & & & $\times$ & $\times$ & & \\
M5 $^{\prime}$ & $\times$ & $\times$ & & & $\times$ & $\times$ & & $\times$ & $\times$ & & \\
\hline M2 & $\times$ & $\times$ & $\times$ & 11 & & & & & \\
\hline
\end{tabular}

FIGURE 4: The dual M-theory picture

type IIA. These D2-branes can be lifted to the M2-branes in Mtheory. To get the required contents of Chern-Simons description of the ABJM theory, the D3-branes are arranged so as to intersect two NS5-branes along the circle. Moreover, the $k$ D5branes are added to this configuration as summarized in the table given in Figure 3.

The $x^{6}$ direction is compact with period $2 \pi R$, with the two NS5-branes located at $x^{6}=0$ and $x^{6}=\pi R . x^{6}=0$ is the locus of D5-branes. Resolving the intersections of the NS5-brane with the $k$ D5-branes produces a $(p, q) 5$-brane web. The resulting theory is super Yang-Mills with massive chiral multiplets. Integrating out the chirality gives rise to the Chern-Simons theory. Finally, the T-duality operation along $x^{6}$ followed by the lift to 11-dimensions gives rise to M2-branes spanning $\left(x^{0}, x^{1}, x^{2}\right)$. Under the T-duality, the 5-branes turn into KK-monopoles and D6-branes. The low-energy description is thus given by M2branes probing $\mathbb{C}^{4} / \mathbb{Z}_{k}$.

In summary, the configuration of $\mathrm{N} \mathrm{D}^{+}$-branes and $\mathrm{N}$ D3 ${ }^{-}$-branes that are stretched between coincident NS5- and NS5'-branes can be lifted to the M-theory configuration M5-N $\mathrm{M} 2-M 5^{\prime}$ to give a GL(N|N) WZW model. In the same way, the configuration figure (Figure 4) M5-N M2-M5'-M M2-M5 will give $\mathrm{GL}(\mathrm{N} \mid \mathrm{N}) \times \mathrm{GL}(\mathrm{M} \mid \mathrm{M})$ WZW model with additional bifundamental matter content.

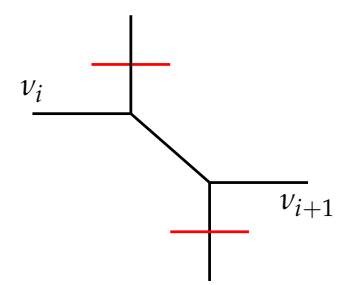

FIGURE 5: Toric diagram for the open string wave function $\mathcal{W}_{v_{i} v_{i+1}}$. Red lines denote the periodicity of the vertical side.

The open topological string wave function $\mathcal{W}_{v_{i} v_{i+1}}$, which is a building block of the partition function (11), is also the counting function for BPS excitations corresponding to the intersecting configurations of M2- and M5- branes. The topologi- 
cal open string wavefunction takes the form [1]

$$
\begin{aligned}
& \mathcal{W}_{v_{i} v_{i+1}}\left(Q_{\tau}, Q_{m}, t, q\right)=t^{-\frac{\| v_{m+1}||^{2}}{2}} q^{-\frac{\left\|v_{m}\right\|^{2}}{2}} \tilde{Z}_{v_{m}^{t}}\left(q^{-1}, t^{-1}\right) \\
& \tilde{Z}_{v_{m+1}}\left(t^{-1}, q^{-1}\right) Q_{m}^{-\frac{\left|v_{m}\right|+\left|v_{m+1}\right|}{2}} \prod_{k=1}\left(1-Q_{\tau}^{k}\right)^{-1} \\
& \times \prod_{i, j=1} \frac{\left(1-Q_{\tau}^{k} Q_{m}^{-1} q^{v_{m+1, i}-j+\frac{1}{2}} t^{v_{m, j}-i+\frac{1}{2}}\right)\left(1-Q_{\tau}^{k-1} Q_{m} q^{v_{m, i}-j+\frac{1}{2}} t^{v_{m+1, j}^{t}-i+\frac{1}{2}}\right)}{\left(1-Q_{\tau}^{k} q^{v_{m+1, i}-j+1} t^{v_{m+1, j}-i}\right)\left(1-Q_{\tau}^{k} q^{v_{m, i}-j} t^{v_{m, j}^{t}-i+1}\right)} .
\end{aligned}
$$

We can rewrite the factor $\mathbf{W}_{\mathbf{k}_{1} \mathbf{k}_{2} \ldots \mathbf{k}_{\mathbf{r}}}$, given in equation (46), in terms of the open topological string wave function $\mathcal{W}_{v_{a} v_{a+1}}$. Using the definition of $Z_{k_{1} k_{2} \ldots k_{r}}$ [4] as

$$
Z_{k_{1} k_{2} \ldots k_{r}}=(-1)^{k_{1}+k_{2}+k_{3}+\ldots k_{n}} \sum_{v_{a},\left|v_{a}\right|=k_{a}} \mathcal{W}_{\varnothing v_{1}} \mathcal{W}_{v_{1} v_{2}} \mathcal{W}_{v_{2} v_{3} \ldots,} \mathcal{W}_{v_{n} \varnothing}
$$

we can write (47) in terms of the topological string wavefunction $\mathcal{W}_{v \mu}$ as

$$
\begin{aligned}
& Z_{k_{1} k_{2} \ldots k_{r}} \varnothing \varnothing \ldots \varnothing \varnothing \ldots\left(\tau, m, \epsilon_{1}, \epsilon_{2}\right) \mathbf{W}_{k_{1} k_{2} \ldots k_{r}}^{2}\left(\tau, m, \epsilon_{1}, \epsilon_{2}\right) \\
& =(-1)^{k_{1}+k_{2}+k_{3}+\ldots k_{3 r}} \sum_{v_{a},\left|v_{a}\right|=k_{a}} \mathcal{W}_{\varnothing v_{1}} \mathcal{W}_{v_{1} v_{2}} \mathcal{W}_{v_{2} v_{3} \ldots} \mathcal{W}_{v_{r-1} v_{r}} \\
& \mathcal{W}_{v_{r+1} v_{r+2} \ldots} \mathcal{W}_{v_{2 r-1} v_{2 r}} \mathcal{W}_{v_{2 r+1} v_{2 r+2} \ldots} \mathcal{W}_{v_{3 r-1} v_{3 r}} \mathcal{W}_{v_{3 r} \varnothing} \\
& \left(\mathcal{W}_{v_{r} v_{r+1}}-\mathcal{W}_{v_{r} \varnothing} \mathcal{W}_{\varnothing v_{r+1}}\right)\left(\mathcal{W}_{v_{2 r} v_{2 r+1}}-\mathcal{W}_{v_{2 r}} \varnothing \mathcal{W}_{\varnothing v_{2 r+1}}\right) .
\end{aligned}
$$

After normalizing by the closed topological string partition function $\mathcal{W}_{\varnothing \varnothing}\left(Q_{\tau}, Q, t, q\right)$, the resulting expression is

$$
\begin{aligned}
& D_{v_{i} v_{i+1}}\left(Q_{\tau}, Q, t, q\right)=\frac{\mathcal{W}_{v_{i} v_{i+1}}\left(Q_{\tau}, Q, t, q\right)}{\mathcal{W}_{\varnothing \varnothing}\left(Q_{\tau}, Q, t, q\right)}=t^{-\frac{\left\|v_{m+1}\right\|^{2}}{2}} q^{-\frac{\left\|v_{m}\right\|^{2}}{2}} \\
& Q_{m}^{-\frac{\left|v_{m}\right|+\left|v_{m+1}\right|}{2}} \prod_{k=1} \prod_{(i, j) \in v_{m}}
\end{aligned}
$$

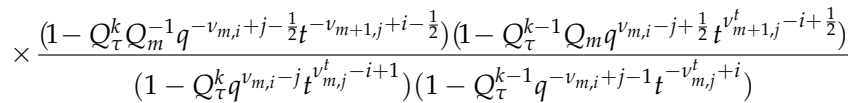

$$
\begin{aligned}
& \times \prod_{(i, j) \in v_{m+1}} \\
& \frac{\left(1-Q_{\tau}^{k} Q_{m}^{-1} q^{v_{m+1, i}-j+\frac{1}{2}} t^{v_{m, j}^{t}-i+\frac{1}{2}}\right)\left(1-Q_{\tau}^{k-1} Q_{m} q^{-v_{m+1, i}+j-\frac{1}{2}} t^{-v_{m, j}^{t}+i-\frac{1}{2}}\right)}{\left(1-Q_{\tau}^{k} q^{v_{m+1, i}-j+1} t^{v_{m+1, j}^{t}}\right)\left(1-Q_{\tau}^{k-1} q^{-v_{m+1, i}+j} t^{-v_{m+1, j}^{t}+i-1}\right)} \\
& =\left(t^{-\frac{\|\left. v_{m+1}\right|^{2}}{2}} Q_{m}^{-\frac{\left|v_{m+1}\right|}{2}} \frac{\left(1-Q_{m} q^{v_{m, i}-j+\frac{1}{2}} t^{v_{m+1, j}^{t}-i+\frac{1}{2}}\right)}{\left(1-q^{-v_{m, i}+j-1} t^{-v_{m, j}^{t}+i}\right)}\right. \\
& \times \prod_{k=1} \prod_{(i, j) \in v_{m}} \\
& \left.\frac{\left(1-Q_{\tau}^{k} Q_{m}^{-1} q^{-v_{m, i}+j-\frac{1}{2}} t^{-v_{m+1, j}+i-\frac{1}{2}}\right)\left(1-Q_{\tau}^{k} Q_{m} q^{v_{m, i}-j+\frac{1}{2}} t^{v_{m+1, j}^{t}-i+\frac{1}{2}}\right)}{\left(1-Q_{\tau}^{k} q^{v_{m, i}-j} t^{v_{m, j}^{t}-i+1}\right)\left(1-Q_{\tau}^{k} q^{-v_{m, i}+j-1} t^{-v_{m, j}^{t}+i}\right)}\right) \\
& \times\left(q^{-\frac{\left\|v_{m}\right\|^{2}}{2}} Q_{m}^{-\frac{\left|v_{m}\right|}{2}} \frac{\left(1-Q_{m} q^{-v_{m+1, i}+j-\frac{1}{2}} t^{-v_{m, j}^{t}+i-\frac{1}{2}}\right)}{\left(1-q^{-v_{m+1, i}+j^{-}} t_{m+1, j}^{t}+i-1\right.}\right) \prod_{k=1} \\
& \times \prod_{(i, j) \in v_{m+1}} \\
& \left.\frac{\left(1-Q_{\tau}^{k} Q_{m}^{-1} q^{v_{m+1, i}-j+\frac{1}{2}} t^{v_{m, j}^{t}-i+\frac{1}{2}}\right)\left(1-Q_{\tau}^{k} Q_{m} q^{-v_{m+1, i}+j-\frac{1}{2}} t^{-v_{m, j}^{t}+i-\frac{1}{2}}\right)}{\left(1-Q_{\tau}^{k} q^{v_{m+1, i}-j+1} t^{v_{m+1, j}^{t}}\right)\left(1-Q_{\tau}^{k} q^{-v_{m+1, i}+j} t^{-v_{m+1, j}^{t}+i-1}\right)}\right) .
\end{aligned}
$$

It can be interpreted [18] as the degrees of freedom of two interacting WZW models. To see this, we consider the first factor of equation (63):

$$
\begin{aligned}
& \left(t^{-\frac{\left\|v_{m+1}\right\|^{2}}{2}} Q_{m}^{-\frac{\left|v_{m+1}\right|}{2}} \frac{\left(1-Q_{m} q^{v_{m, i}-j+\frac{1}{2}} t^{v_{m+1, j}^{t}-i+\frac{1}{2}}\right)}{\left(1-q^{-v_{m, i}+j-1} t^{-v_{m, j}^{t}+i}\right)} \prod_{k=1} \prod_{(i, j) \in v_{m}}\right. \\
& \left.\frac{\left(1-Q_{\tau}^{k} Q_{m}^{-1} q^{-v_{m, i}+j-\frac{1}{2}} t^{-v_{m+1, j}+i-\frac{1}{2}}\right)\left(1-Q_{\tau}^{k} Q_{m} q^{v_{m, i}-j+\frac{1}{2}} t^{v_{m+1, j}^{t}-i+\frac{1}{2}}\right)}{\left(1-Q_{\tau}^{k} q^{v_{m, i}-j} t^{v_{m, j}^{t}-i+1}\right)\left(1-Q_{\tau}^{k} q^{-v_{m, i}+j-1} t^{-v_{m, j}^{t}+i}\right)}\right) .
\end{aligned}
$$

This factor is identical to the contribution of the field content of a supersymmetric WZW model [19, 20, 21].

The expression (64) is reminiscent of the $\mathcal{N}=2$ minimal model product representation of the elliptic genus [19]. It was suggested and proved in [20, 21, 22] that the minimal models are equivalent to super-renormalizable Landau-Ginzburg models in the sense that the latter flows to the former at UV conformal point. To this end, we use the known characters of the discrete series representation of the $N=2$ superconformal algebra to compute its elliptic genus. For the Landau-Ginzburg model, a certain superpotential deformation was used to render the exact computation of the elliptic genus possible.

It is desirable to independently compute the elliptic genus of the minimal model using some Lagrangian formulation of it. It turned out that certain minimal models have Lagrangian description as supersymmetric gauged WZW models. The equivalence was shown by demonstrating that the elliptic genus computed for a Landau-Ginzburg model matches the elliptic genus computed for a particular supersymmetric gauged WZW model [21].

The field content of the supersymmetric gauged WZW model comprises [21] a Lie group $G$ valued bosonic field $g$, a gauge field $A_{\alpha}$ that is Lie(H)-valued, where $\mathrm{H} \subset \mathrm{G}$ and $\mathrm{Lie}(\mathrm{G} / \mathrm{H})$ valued left moving and right moving fermionic fields $\widetilde{\psi}_{+}$and $\widetilde{\psi}_{-}$, respectively. The dynamics is given by the Lagrangian

$$
\begin{aligned}
S= & -\frac{k}{8 \pi} \int d^{2} z \sqrt{h} h^{i j} \operatorname{Trg}^{-1} \partial_{i} g \cdot g^{-1} \partial_{j} g \\
& -\frac{i k}{12 \pi} \int_{B} d^{2} \sigma \epsilon^{i j k} \operatorname{Tr}^{-1} \partial_{i} g \cdot g^{-1} \partial_{j} g \cdot g^{-1} \partial_{k} g \\
& +\frac{k}{2 \pi} \int d^{2} z \operatorname{Tr}\left(A_{\bar{z} g^{-1}} \partial_{z} g-A_{z} \partial_{\bar{z} g g^{-1}}\right. \\
& \left.-A_{\bar{z}} A_{z}+A_{\bar{z}} g^{-1} A_{z} g\right) \\
& +\frac{i k}{4 \pi} \int d^{2} z \operatorname{Tr}\left(\widetilde{\psi}_{+} D_{\bar{z}} \widetilde{\psi}_{+}+\widetilde{\psi}_{-} D_{z} \widetilde{\psi}_{-}\right),
\end{aligned}
$$

where $\mathrm{B}$ is the 3-manifold whose boundary is the $2 \mathrm{~d}$ worldsheet, $h_{i j}$ is the worldsheet metric, $D_{z}=\partial_{z}+\left[A_{z}\right.$, $]$ and $D_{\bar{z}}=$ $\partial_{\bar{z}}+\left[A_{\bar{z}},\right]$ are the covariant derivatives, and the integer $k$ is the level. After the identification of a global $U(1)$ that is part of the left moving $N=2$ algebra, the charge assignment of the fields is given by

$$
\begin{aligned}
\widetilde{\psi}_{+} & \rightarrow e^{i \frac{\gamma}{(k+2)} \widetilde{\psi}_{+}} \\
\widetilde{\psi}_{-} & \rightarrow e^{i \frac{\gamma(k+1)}{(k+2)} \widetilde{\psi}_{-}} \\
g & \rightarrow-i \frac{\gamma}{(k+2)}(U g+g U) \\
A_{\alpha} & \rightarrow A_{\alpha},
\end{aligned}
$$

where $\mathrm{U} \in \mathrm{Lie} \mathrm{SU}(2)$ denotes the generator of the $\mathrm{U}(1) \subset \mathrm{SU}(2)$ which is gauged. This allows us to show that the elliptic genus 
of the supersymmetric WZW model of the coset $\mathrm{SU}(2) / \mathrm{U}(1)$ is given by

$$
\begin{aligned}
\mathcal{E}^{S W Z W}= & e^{-i \gamma k \alpha / 2} \frac{1-e^{i \gamma(k+1) \alpha}}{1-e^{i \gamma \alpha}} \\
& \times \prod_{n=1}^{\infty} \frac{\left(1-q^{n} e^{i \gamma(k+1) \alpha}\right)\left(1-q^{n} e^{-i \gamma(k+1) \alpha}\right)}{\left(1-e^{i \gamma \alpha}\right)\left(1-e^{-i \gamma \alpha}\right)},
\end{aligned}
$$

where the contribution of the fermionic zero modes is separated, respectively, as

$$
\frac{1}{\left(1-e^{i \gamma \alpha}\right)\left(1-e^{-i \gamma \alpha}\right)}
$$

and bosonic zero modes as

$$
e^{-i \gamma k \alpha / 2}\left(1-e^{i \gamma(k+1) \alpha}\right)\left(1-e^{-i \gamma \alpha}\right) .
$$

Moreover, the nonzero mode contribution of the fermions is separated as

$$
\left(1-q^{n} e^{i \gamma(k+1) \alpha}\right)\left(1-q^{n} e^{-i \gamma(k+1) \alpha}\right)\left(1-\bar{q}^{n} e^{i \gamma \alpha}\right)\left(1-\bar{q}^{n} e^{-i \gamma \alpha}\right)
$$

and the bosons as

$$
\frac{1}{\left(1-q^{n} e^{i \gamma \alpha}\right)\left(1-q^{n} e^{-i \gamma \alpha}\right)\left(1-\bar{q}^{n} e^{i \gamma \alpha}\right)\left(1-\bar{q}^{n} e^{-i \gamma \alpha}\right)} .
$$

Note that the antiholomorphic part is canceled out.

The various contributions of the supersymmetric WZW model are thus arranged in the following.

- Contribution of the Fermionic and Bosonic Zero Modes:

$$
\frac{\left(1-Q_{m} q^{v_{m, i}-j+\frac{1}{2}} t^{v_{m+1, j}^{t}-i+\frac{1}{2}}\right)}{\left(1-q^{-v_{m, i}+j-1} t^{-v_{m, j}^{t}+i}\right)}
$$

- Contribution of the Fermionic and Bosonic Nonzero Modes:

$$
\begin{aligned}
& \left(\prod_{k=1} \prod_{(i, j) \in v_{m}}\right. \\
& \frac{\left(1-Q_{\tau}^{k} Q_{m}^{-1} q^{-v_{m, i}+j-\frac{1}{2}} t^{-v_{m+1, j}+i-\frac{1}{2}}\right)\left(1-Q_{\tau}^{k} Q_{m} q^{v_{m, i}-j+\frac{1}{2}} t^{v_{m+1, j}^{t}-i+\frac{1}{2}}\right)}{\left(1-Q_{\tau}^{k} q^{v_{m, i}-j} t^{v_{m, j}^{t}-i+1}\right)\left(1-Q_{\tau}^{k} q^{-v_{m, i}+j-1} t^{-v_{m, j}^{t}+i}\right)} .
\end{aligned}
$$

- Contribution from the Phase Factors:

$$
\left(t^{-\frac{\left\|v_{m+1}\right\|^{2}}{2}} Q_{m}^{-\frac{\left|v_{m+1}\right|}{2}}\right)
$$

Note that for the second WZW model the phase contribution changes from $t^{-\frac{\left\|v_{m+1}\right\|^{2}}{2}}$ to $q^{-\frac{\left\|v_{m}\right\|^{2}}{2}}$, i.e., from a $t$-factor to a $q$ factor. Similarly, the second factor in equation (63) also describes a WZW model.

Recall that the partition function $Z_{N}\left(\tau, m, t_{f_{1}}, t_{f_{2}}, \ldots, t_{f_{N}}\right.$, $\left.\epsilon_{1}, \epsilon_{2}\right)$ of $N$ parallel and separated M5-branes with M2-branes stretched between them can alternatively [1] be written in terms of the normalised open topological string wavefunctions $D_{v_{i} v_{i+1}}\left(Q_{\tau}, Q, t, q\right)$ as

$$
\begin{aligned}
& Z_{N}\left(\tau, m, t_{f_{1}}, t_{f_{2}}, \ldots, t_{f_{N}}, \epsilon_{1}, \epsilon_{2}\right) \\
& =\sum_{v_{1}, \ldots, v_{N-1}}\left(\prod_{a=1}^{N-1}\left(-Q_{f_{a}}\right)^{\left|v_{a}\right|}\right) \times D_{\varnothing v_{1}}\left(Q_{\tau}, Q, t, q\right) \\
& \quad \times D_{v_{1} v_{2}}\left(Q_{\tau}, Q, t^{-1}, q^{-1}\right) D_{v_{2} v_{3}}\left(Q_{\tau}, Q, t, q\right) \ldots D_{v_{N-1}} \varnothing .
\end{aligned}
$$

This form of the partition function allows an interpretation in terms of $N$ domain walls interpolating between the M2-brane vacua. In terms of the supersymmetric WZW model, we can say that the partition function is a superposition of the wavefunctions of a chain of coupled supersymmetric WZW models. The center of mass motion of the multiple M-strings as well as their mutual dynamics is encoded in this wave function. For example, for the case of two M-strings, it involves their center of mass motion as well as their motion relative to each other. The components of the elliptic genus $Z_{v_{1} v_{2}, \ldots, v_{k}}$ are related to the open topological string wave function. For example,

$$
\begin{aligned}
Z_{22}-Z_{2 \varnothing}^{2} & =\mathcal{W}_{\varnothing 2} \mathcal{W}_{2 \varnothing}\left(\mathcal{W}_{22}-\mathcal{W}_{\varnothing 2} \mathcal{W}_{2 \varnothing}\right), \\
Z_{33}-Z_{3 \varnothing}^{2} & =\mathcal{W}_{\varnothing 3} \mathcal{W}_{3 \varnothing}\left(\mathcal{W}_{33}-\mathcal{W}_{\varnothing 3} \mathcal{W}_{3 \varnothing}\right), \\
Z_{1212}-Z_{12 \varnothing \varnothing}^{2} & =\mathcal{W}_{\varnothing 1} \mathcal{W}_{12} \mathcal{W}_{2 \varnothing}\left(\mathcal{W}_{21} \mathcal{W}_{12}-\mathcal{W}_{\varnothing 1} \mathcal{W}_{12} W_{2 \varnothing}\right) .
\end{aligned}
$$

In other words, the universal factors $W_{2}\left(\tau, m, \epsilon_{1}, \epsilon_{2}\right), W_{3}(\tau, m$, $\left.\epsilon_{1}, \epsilon_{2}\right)$ and $W_{12}\left(\tau, m, \epsilon_{1}, \epsilon_{2}\right)$ for these M5-M2 brane configurations can be expressed in terms of open topological wavefunction in equation (60) as

$$
\begin{aligned}
W_{2}\left(\tau, m, \epsilon_{1}, \epsilon_{2}\right) & =\left(\mathcal{W}_{22}-\mathcal{W}_{\varnothing 2} \mathcal{W}_{2 \varnothing}\right), \\
W_{3}\left(\tau, m, \epsilon_{1}, \epsilon_{2}\right) & =\left(\mathcal{W}_{33}-\mathcal{W}_{\varnothing 3} \mathcal{W}_{3 \varnothing}\right), \\
W_{12}\left(\tau, m, \epsilon_{1}, \epsilon_{2}\right) & =\left(\mathcal{W}_{21} \mathcal{W}_{12}-\mathcal{W}_{\varnothing 1} \mathcal{W}_{12} W_{2 \varnothing}\right) .
\end{aligned}
$$

Similarly, we can write for $W_{N}\left(\tau, m, \epsilon_{1}, \epsilon_{2}\right)$

$$
W_{N}\left(\tau, m, \epsilon_{1}, \epsilon_{2}\right)=\left(\mathcal{W}_{N N}-\mathcal{W}_{\varnothing N} \mathcal{W}_{N \varnothing}\right)
$$

Recall that in a given M-theory vacuum the coupling constant $\tau$ is related to the radius of the circle $S^{1}$ parallel to the M5brane worldvolume. Formally, we can consider different coupling constants $\tau_{i}$ for different domain walls. Each $\tau_{i}$ is related to the circle $S^{1}$ parallel to the $i$-th M5-brane worldvolume. This M-theory setup can be dualized in type IIB strings to a $5 \mathrm{~d} \mathcal{N}=1^{*}$ supersymmetric gauge theory living on a particular $(p, q)$ D5-NS5-brane web. $\tau_{i}$ correspond to the gauge coupling constant of the supersymmetric gauge theories dual to corresponding M5 brane-M2 brane-M-string configurations. For these general cases, for instance, we can write

$$
\begin{aligned}
& Z_{1212}-Z_{12 \varnothing \varnothing} Z_{\varnothing 12 \varnothing} \\
& =\mathcal{W}_{\varnothing 1}\left(\tau_{1}\right) \mathcal{W}_{12}\left(\tau_{4}\right) \mathcal{W}_{2 \varnothing}\left(\tau_{5}\right) \\
& \times\left(\mathcal{W}_{12}\left(\tau_{2}\right) \mathcal{W}_{21}\left(\tau_{3}\right)-\mathcal{W}_{\varnothing 1}\left(\tau_{3}\right) \mathcal{W}_{12}\left(\tau_{2}\right) \mathcal{W}_{2 \varnothing}\left(\tau_{3}\right)\right. \\
& Z_{222}-Z_{22 \varnothing} Z_{\varnothing \varnothing 2}-Z_{\varnothing 22} Z_{2 \varnothing \varnothing}-Z_{2 \varnothing \varnothing} Z_{\varnothing 2 \varnothing} Z_{\varnothing \varnothing 2} \\
& =\mathcal{W}_{\varnothing 2}\left(\tau_{1}\right) \mathcal{W}_{2 \varnothing}\left(\tau_{4}\right) \\
& \quad \times\left(\left(\mathcal{W}_{22}\left(\tau_{2}\right)-\mathcal{W}_{\varnothing 2}\left(\tau_{2}\right) \mathcal{W}_{2 \varnothing}\left(\tau_{2}\right)\right)\right. \\
& \left.\quad \times\left(\mathcal{W}_{22}\left(\tau_{3}\right)-\mathcal{W}_{\varnothing 2}\left(\tau_{3}\right) \mathcal{W}_{2 \varnothing}\left(\tau_{3}\right)\right)\right)
\end{aligned}
$$


More generally, we can write for $v_{1}=v_{2}=v_{3}$ the recursive relation for $Z_{v_{1} v_{2} v_{2}}$ as

$$
\begin{aligned}
& Z_{v_{1} v_{2} v_{3}}-Z_{v_{1} v_{2}} Z_{v_{3}}-Z_{v_{1}} Z_{v_{2} v_{3}}+Z_{v_{1}} Z_{v_{2}} Z_{v_{3}} \\
& \quad=\mathcal{W}_{\varnothing v_{1}} \mathcal{W}_{\varnothing v_{3}}\left(\mathcal{W}_{v_{1} v_{2}}-\mathcal{W}_{\varnothing v_{1}} \mathcal{W}_{\varnothing v_{2}}\right)\left(\mathcal{W}_{v_{2} v_{3}}-\mathcal{W}_{\varnothing v_{2}} \mathcal{W}_{\varnothing v_{3}}\right)
\end{aligned}
$$

Comparing the last expression with equation (20), we see that the first factor $\mathcal{W}_{\varnothing v_{1}} \mathcal{W}_{\varnothing v_{3}}$ is the result of fusing all the partitions, the second factor $\left(\mathcal{W}_{v_{1} v_{2}}-\mathcal{W}_{\varnothing v_{1}} \mathcal{W}_{\varnothing v_{2}}\right)$ appears when we fuse the partitions $v_{1}, v_{2}$ along with the removal of the second M5-brane, and the third factor $\left(\mathcal{W}_{v_{2} v_{3}}-\mathcal{W}_{\varnothing v_{2}} \mathcal{W}_{\varnothing v_{3}}\right)$ appears when we remove the third M5-brane fusing $v_{2}, v_{3}$.

A generalization of $Z_{v_{1} v_{2} v_{3} v_{4} \ldots v_{k}}$ for $v_{1}=v_{2}=v_{3}=\ldots=v_{k}$ can be expressed as

$$
\begin{aligned}
Z_{v_{1} v_{2} v_{3} v_{4} \ldots v_{k}}-Z_{v_{1} v_{2} v_{3} \ldots v_{k-1}} Z_{v_{k}}-Z_{v_{2} v_{3} v_{4} \ldots v_{k}} Z_{v_{1}}+Z_{v_{1}} Z_{v_{2}} Z_{v_{3} v_{4} \ldots v_{k}} \\
\quad+Z_{v_{k-1}} Z_{v_{k}} Z_{v_{1} v_{2} v_{3} v_{4} \ldots v_{k-2}}+\ldots-Z_{v_{1}} Z_{v_{2}} Z_{v_{3}} \ldots Z_{v_{k}} \\
=\mathcal{W}_{\varnothing v_{1}} \mathcal{W}_{\varnothing v_{4}}\left(\mathcal{W}_{v_{1} v_{2}}-\mathcal{W}_{\varnothing v_{1}} \mathcal{W}_{\varnothing v_{2}}\right)\left(\mathcal{W}_{v_{2} v_{3}}-\mathcal{W}_{\varnothing v_{2}} \mathcal{W}_{\varnothing v_{3}}\right) \\
\quad \times\left(\mathcal{W}_{v_{3} v_{4}}-\mathcal{W}_{\varnothing v_{3}} \mathcal{W}_{\varnothing v_{4}}\right) \ldots\left(\mathcal{W}_{v_{k-1} v_{k}}-\mathcal{W}_{\varnothing v_{k-1}} \mathcal{W}_{\varnothing v_{k}}\right) .
\end{aligned}
$$

\section{ABJM MODEL VS M-THEORY}

The ABJM model is defined by a $3 \mathrm{~d} \mathcal{N}=6$ supersymmetric $U(N)_{k} \times U(N)_{-k}$ Chern-Simons theory with matter coupling given by the bifundamental scalars $Z_{a}$ and spinors $\Psi^{a}$ with SU(4) R-symmetry index $a$. The low-energy $2 d$ gauge theory corresponds to the reduction of the worldvolume theory of M2-branes to two dimensions with the boundary conditions provided by the M5-branes. For details, we refer the reader to [23]. This 2d theory is termed as ABJM slab and is identical to the $\mathcal{N}=(4,4)$ super Yang-Mills having $S U(2)^{3}$ R-symmetry, and the gauge coupling $g_{2 d}^{2}$ is determined by the distance between the M2-branes stack and the $\mathbb{Z}_{k}$ orbifold singularity of the transverse space $\mathbb{C}^{2} / \mathbb{Z}_{k}$. This $2 \mathrm{~d}$ theory is special in the sense that the M2-branes do not sit on top of the $\mathbb{Z}_{k}$ singularity. This avoids the appearance of Nahm poles. Moreover, the Ramond-Ramond boundary conditions used in the definition of elliptic genus project out the massive modes corresponding to the KK modes. The elliptic genus of the $2 \mathrm{~d}$ gauge theory with nonzero coupling constant $g_{2 d}^{2}>0$ turns out to be the same as that of $\mathcal{N}=(4,4)$ super Yang-Mills.

The M2-M5 brane intersection is described by the boundary conditions that preserve six supercharges and the $S U(2) \times$ $S U(2) \times U(1)$ subgroup of the full R-symmetry group $S U(4)$. The two scalars $Z_{1}$ and $Z_{2}$ are longitudinal to the M5-brane and form a doublet under one of the two $S U(2) s$. The other two scalars $Z_{3}$ and $Z_{4}$ are transverse and form a doublet under the second $S U(2)$. Moreover, under the $U(1)$ group, the two doublets are oppositely charged. The boundary conditions on bosons and fermions are given as

$$
\begin{gathered}
\Psi_{+}^{1}=\Psi_{+}^{2}=\Psi_{-}^{1}=\Psi_{-}^{2}=0, \quad \bar{\Psi}_{+}^{1}=\bar{\Psi}_{+}^{2}=\bar{\Psi}_{-}^{1}=\bar{\Psi}_{-}^{2}=0, \\
D_{\mu} Z_{A}=0, \quad D_{x_{2}} Z_{I}=\frac{2 \pi}{k}\left(Z_{I} \bar{Z}^{J} Z_{J}-Z_{J} \bar{Z}^{J} Z_{I}\right), \\
Z_{A} \bar{Z}^{I} Z_{B}=Z_{B} \bar{Z}^{I} Z_{A}, \quad Z_{I} \bar{Z}^{A} Z_{B}=Z_{B} \bar{Z}^{A} Z_{I} .
\end{gathered}
$$

The boundary forces the gauge fields of the two $U(N) s$ to be related as

$$
F_{\mu v} Z_{A}=Z_{A} \tilde{F}_{\mu v}
$$

Moreover, the variation of the ABJM action gives rise to a boundary term

$$
\delta S=\frac{k}{4 \pi} \int_{\text {boundary }} \operatorname{Tr}(\alpha d A-\tilde{\alpha} d \tilde{A})
$$

which vanishes only if $A=\tilde{A}$ and $\alpha=\tilde{\alpha}$. However, if one takes $A \neq \tilde{A}$, then the anomalous boundary term can be canceled by introducing boundary fermions of one chirality coupled to $A$ and boundary fermions of the opposite chirality coupled to $\tilde{A}$. This effectively gives rise to WZW model degrees of freedom at the boundary and the gauge anomaly they generate cancels the anomalous term (85).

We first write down the expression of the elliptic genus of the $2 \mathrm{~d}$ gauge theory obtained from the dimensional reduction of the ABJM model for the case $k=1$ as considered in [23]

$$
\begin{aligned}
& Z_{\mathrm{T}^{2}}^{A B J M} \\
& =\int \prod_{i=1}^{N} \frac{d w_{i} d \bar{w}_{i}}{\operatorname{Im} \tau} \prod_{i, j} \frac{\theta_{1}\left(w_{i}-w_{j}+m+\epsilon_{+}\right) \theta_{1}\left(w_{i}-w_{j}+m-\epsilon_{+}\right)}{\theta_{1}\left(w_{i}-w_{j}+\epsilon_{1}\right) \theta_{1}\left(w_{i}-w_{j}+\epsilon_{2}\right)} .
\end{aligned}
$$

However, in our case, the integral is finite with respect to the integration variables, and no special regularization is required. We will use instead a prescription given in [24] for the case of $N=2$.

For $\mathrm{ABJM}$ theory and for $N=1$, we get the expression

$$
\mathrm{Z}_{\mathrm{T}^{2}}^{A B J M}=\frac{\theta_{1}\left(m+\epsilon_{+}\right) \theta_{1}\left(m-\epsilon_{+}\right)}{\theta_{1}\left(\epsilon_{1}\right) \theta_{1}\left(\epsilon_{2}\right)} .
$$

This expression matches with its $Z_{T^{2}}^{M-\text { string }}$ [1].

$N \geq 2$

For the complex integration beyond and including $N=2$, we will use the results $[24,25,26]$

$$
\begin{aligned}
& Z_{\mathrm{T}^{2}}^{A B J M}=\left(\frac{\theta_{1}\left(m+\epsilon_{+}\right) \theta_{1}\left(m-\epsilon_{+}\right)}{\theta_{1}\left(\epsilon_{1}\right) \theta_{1}\left(\epsilon_{2}\right)}\right)^{N} \\
& \int \prod_{i=1}^{N} \frac{d w_{i} d \bar{w}_{i}}{\operatorname{Im\tau }} \prod_{j \neq i} \frac{\theta_{1}\left(w_{i}-w_{j}+m+\epsilon_{+}\right) \theta_{1}\left(w_{i}-w_{j}+m-\epsilon_{+}\right)}{\theta_{1}\left(w_{i}-w_{j}+\epsilon_{1}\right) \theta_{1}\left(w_{i}-w_{j}+\epsilon_{2}\right)} .
\end{aligned}
$$

The $w_{i}, \bar{w}_{i}$ integrals are well defined and do not require any regularization. So we can write normalized elliptic genus in the limit $Q_{m}=\sqrt{\frac{t}{q}}$ as

$$
\widehat{Z}_{\mathrm{T}^{2}}^{A B M}=\int \prod_{i=1}^{N} \frac{d z_{i}}{2 \pi i z_{i}} \prod_{j \neq i} \frac{\theta_{1}\left(\frac{z_{i}}{z_{j}} \frac{t}{q}\right) \theta_{1}\left(\frac{z_{i}}{z_{j}}\right)}{\theta_{1}\left(\frac{z_{i}}{z_{j}} t\right) \theta_{1}\left(\frac{z_{i}}{z_{j}} q^{-1}\right)} .
$$

For $2 \mathrm{~d}$ gauge theories containing the adjoint matter, the poles contributing to the elliptic genus were found to be $[25,26]$

$$
z_{a}=t^{x} q^{-y}
$$


where $(x, y)$ are the coordinates of the $a$-th box in the Young diagram $\mu$ such that $|\mu|=N$ for all $N \geq 0$. Evaluating the residue of (89) on these poles, we get

$$
\begin{aligned}
& \widehat{Z}_{\mathrm{T}^{2}}^{A B J M}=\sum \prod_{\left(x_{1}, y_{1}\right) \in Y,\left(x_{2}, y_{2}\right) \in Y^{t}}^{x 1 \neq x_{2}, y_{1} \neq y_{2}} \\
& \frac{\theta_{1}\left(\left(x_{1}-x_{2}\right) \epsilon_{1}+\left(y_{1}-y_{2}\right) \epsilon_{2}\right) \theta_{1}\left(\left(x_{1}-x_{2}-1\right) \epsilon_{1}+\left(y_{1}-y_{2}-1\right) \epsilon_{2}\right)}{\theta_{1}\left(\left(x_{1}-x_{2}-1\right) \epsilon_{1}+\left(y_{1}-y_{2}\right) \epsilon_{2}\right) \theta_{1}\left(\left(x_{1}-x_{2}\right) \epsilon_{1}+\left(y_{1}-y_{2}+1\right) \epsilon_{2}\right)} \\
& =\prod \frac{\theta_{1}\left(\epsilon_{1}\right) \theta_{1}\left(\epsilon_{2}\right)}{\theta_{1}^{\prime} \theta_{1}\left(\epsilon_{1}+\epsilon_{2}\right)} \sum_{\left(x_{1}, y_{1}\right) \in Y,\left(x_{2}, y_{2}\right) \in Y^{t}} \prod_{\theta_{1}\left(\left(x_{1}-x_{2}\right) \epsilon_{1}+\left(y_{1}-y_{2}\right) \epsilon_{2}\right) \theta_{1}\left(\left(x_{1}-x_{2}-1\right) \epsilon_{1}+\left(y_{1}-y_{2}-1\right) \epsilon_{2}\right)} \\
& \frac{\theta_{1}\left(\left(x_{1}-x_{2}-1\right) \epsilon_{1}+\left(y_{1}-y_{2}\right) \epsilon_{2}\right) \theta_{1}\left(\left(x_{1}-x_{2}\right) \epsilon_{1}+\left(y_{1}-y_{2}+1\right) \epsilon_{2}\right)}{}
\end{aligned}
$$

This can be written in the canonical form by using the theorem (2.11) of reference [27]:

$$
\begin{aligned}
& \sum_{\left(x_{1}, y_{1}\right) \in Y} t_{1}^{x_{1}} t_{2}^{y_{1}}+\sum_{\left(x_{2}, y_{2}\right) \in Y^{t}} t_{1}^{1-x_{2}} t_{2}^{1-y_{2}} \\
& -\left(\sum_{\left(x_{1}, y_{1}\right) \in Y} t_{1}^{x_{1}-\lambda_{y_{1}}^{t}\left(Y^{t}\right)} t_{2}^{1-y_{1}+\lambda_{x_{1}}(Y)}\right. \\
& \left.\quad+\sum_{\left(x_{2}, y_{2}\right) \in Y^{t}} t_{1}^{1-x_{2}+\lambda_{y_{2}}^{t}(Y)} t_{2}^{y_{2}-\lambda_{x_{2}}\left(Y^{t}\right)}\right) \\
& =\sum_{\left(x_{1}, y_{1}\right) \in Y,\left(x_{2}, y_{2}\right) \in Y^{t}} t_{1}^{x_{1}-x_{2}} t_{2}^{y_{1}-y_{2}}\left(1-t_{1}\right)\left(1-t_{2}\right)
\end{aligned}
$$

as

$$
\begin{aligned}
& \widehat{Z}_{\mathrm{T}^{2}}^{A B J M} \\
& =\prod \frac{\theta_{1}\left(\epsilon_{1}\right) \theta_{1}\left(\epsilon_{2}\right)}{\theta_{1}^{\prime} \theta_{1}\left(\epsilon_{1}+\epsilon_{2}\right)} \sum_{Y, Y^{t}} \prod_{\left(x_{1}, y_{1}\right) \in Y,\left(x_{2}, y_{2}\right) \in Y^{t}} \\
& \frac{\theta_{1}\left(x_{1} \epsilon_{1}-y_{1} \epsilon_{2}\right) \theta_{1}\left(\left(1-x_{2}\right) \epsilon_{1}-\left(1-y_{2}\right) \epsilon_{2}\right)}{\theta_{1}\left(\left(x_{1}-\lambda_{y_{1}}^{t}\right) \epsilon_{1}-\left(1-y_{1}+\lambda_{x_{1}}\right) \epsilon_{2}\right) \theta_{1}\left(\left(1-x_{2}-\lambda_{y_{2}}^{t}\right) \epsilon_{1}-\left(y_{2}-\lambda_{x_{2}}\right) \epsilon_{2}\right)} .
\end{aligned}
$$

It is interesting to compare $\widehat{Z}_{\mathrm{T}^{2}}$ with $\mathrm{Z}_{\mathrm{T}^{2}}^{\text {IIA }}$ given by

$$
\begin{aligned}
Z_{\mathrm{T}^{2}}^{I I A}= & \sum_{Y, Y^{t}} \prod_{(i, j) \in Y} \\
& \times \frac{\theta_{1}\left((i-1) \epsilon_{1}+(j-1) \epsilon_{2}\right) \theta_{1}\left(-i \epsilon_{1}-j \epsilon_{2}\right)}{\theta_{1}\left(\left(i-\lambda_{j}^{t}\right) \epsilon_{1}+\left(\lambda_{i}-j+1\right) \epsilon_{2}\right) \theta_{1}\left(\left(1-i+\lambda_{j}^{t}\right) \epsilon_{1}+\left(j-\lambda_{i}\right) \epsilon_{2}\right)} .
\end{aligned}
$$

\section{CONCLUSIONS}

We have studied the structure of the free energies of M-strings. An interesting recursive structure in the free energies (BPS counting functions) was observed [4] for the configuration -M2-M5-M2-M5-M2- of M2-M5 branes. We show that for configurations containing multiple M2-branes sandwiched between M5-branes the recursive structure in free energies is lost. Instead, the coefficients $Z_{A_{1} A_{2} \ldots A_{n}}$ in the expansion of partition function enjoy the recursive structure. For completeness, we also describe the M2-brane configurations with symmetric representations and mixed representations.

The partition functions of M2-brane configuration that enter the M-strings elliptic genera can also be interpreted as the vacuum-n to vacuum-(n+1) amplitude with M5-branes acting as the domain wall. The M5-brane domain wall acts as the duality transformation that interpolates between the two vacua [8]. ABJM formulation of M2-brane theories allows a more direct study of the domain wall partition functions. We compute the elliptic genus of a dimensionally reduced $2 d$ theory of ABJM slab model and compare it with the M-string computations. We find an interesting mismatch that can be explained in terms of the center of mass motion of M2-M5 branes in the transverse space. The factor corresponding to the mismatch accounts for this center of mass motion in the transverse space.

It will be interesting to study the WZW-topological string correspondence for more general backgrounds in M-theory.

\section{Appendix A. DENOMINATOR FACTORS $D_{v_{1}, \ldots, \nu_{K}}\left(\tau, M, \epsilon_{1}, \epsilon_{2}\right)$}

$$
\begin{aligned}
& D_{134134134}\left(\tau, m, \epsilon_{1}, \epsilon_{2}\right) \\
& =\theta_{1}\left(\epsilon_{1}-m\right)^{9} \theta_{1}\left(-m-4 \epsilon_{2}\right)^{3} \theta_{1}\left(-m-3 \epsilon_{2}\right)^{6} \\
& \theta_{1}\left(-m+\epsilon_{1}-3 \epsilon_{2}\right)^{3} \theta_{1}\left(-m-2 \epsilon_{2}\right)^{6} \times \theta_{1}\left(-m+\epsilon_{1}-2 \epsilon_{2}\right)^{6} \\
& \theta_{1}\left(-m-\epsilon_{2}\right)^{9} \theta_{1}\left(-m+\epsilon_{1}-\epsilon_{2}\right)^{6} \text {, }
\end{aligned}
$$

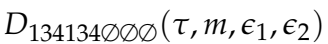

$$
\begin{aligned}
& =\theta_{1}\left(\epsilon_{1}-m\right)^{6} \theta_{1}\left(-m-4 \epsilon_{2}\right)^{2} \theta_{1}\left(-m-3 \epsilon_{2}\right)^{4} \\
& \theta_{1}\left(-m+\epsilon_{1}-3 \epsilon_{2}\right)^{2} \theta_{1}\left(-m-2 \epsilon_{2}\right)^{4} \times \theta_{1}\left(-m+\epsilon_{1}-2 \epsilon_{2}\right)^{4} \\
& \theta_{1}\left(-m-\epsilon_{2}\right)^{6} \theta_{1}\left(-m+\epsilon_{1}-\epsilon_{2}\right)^{4} \text {, }
\end{aligned}
$$

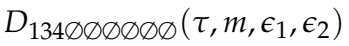

$$
\begin{aligned}
& =\theta_{1}\left(\epsilon_{1}-m\right)^{3} \theta_{1}\left(-m-4 \epsilon_{2}\right) \theta_{1}\left(-m-3 \epsilon_{2}\right)^{2} \\
& \theta_{1}\left(-m+\epsilon_{1}-3 \epsilon_{2}\right) \theta_{1}\left(-m-2 \epsilon_{2}\right)^{2} \times \theta_{1}\left(-m+\epsilon_{1}-2 \epsilon_{2}\right)^{2} \\
& \theta_{1}\left(-m-\epsilon_{2}\right)^{3} \theta_{1}\left(-m+\epsilon_{1}-\epsilon_{2}\right)^{2} \text {, } \\
& D_{232323}\left(\tau, m, \epsilon_{1}, \epsilon_{2}\right) \\
& =\theta_{1}\left(\epsilon_{1}-m\right)^{6} \theta_{1}\left(-m-3 \epsilon_{2}\right)^{3} \theta_{1}\left(-m-2 \epsilon_{2}\right)^{6} \\
& \theta_{1}\left(-m+\epsilon_{1}-2 \epsilon_{2}\right)^{3} \times \theta_{1}\left(-m-\epsilon_{2}\right)^{6} \\
& \theta_{1}\left(-m+\epsilon_{1}-\epsilon_{2}\right)^{6} \text {, }
\end{aligned}
$$

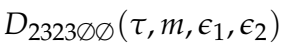

$$
\begin{aligned}
& =\theta_{1}\left(\epsilon_{1}-m\right)^{4} \theta_{1}\left(-m-3 \epsilon_{2}\right)^{2} \theta_{1}\left(-m-2 \epsilon_{2}\right)^{4} \\
& \theta_{1}\left(-m+\epsilon_{1}-2 \epsilon_{2}\right)^{2} \times \theta_{1}\left(-m-\epsilon_{2}\right)^{4} \\
& \theta_{1}\left(-m+\epsilon_{1}-\epsilon_{2}\right)^{4} \text {, } \\
& D_{232323}\left(\tau, m, \epsilon_{1}, \epsilon_{2}\right) \\
& =\theta_{1}\left(\epsilon_{1}-m\right)^{2} \theta_{1}\left(-m-3 \epsilon_{2}\right) \theta_{1}\left(-m-2 \epsilon_{2}\right)^{2} \\
& \theta_{1}\left(-m+\epsilon_{1}-2 \epsilon_{2}\right) \times \theta_{1}\left(-m-\epsilon_{2}\right)^{2} \\
& \theta_{1}\left(-m+\epsilon_{1}-\epsilon_{2}\right)^{2} \text {, } \\
& D_{121212}\left(\tau, m, \epsilon_{1}, \epsilon_{2}\right)=\theta_{1}\left(\epsilon_{1}-m\right)^{6} \theta_{1}\left(-m-2 \epsilon_{2}\right)^{3} \\
& \theta_{1}\left(-m-\epsilon_{2}\right)^{6} \theta_{1}\left(-m+\epsilon_{1}-\epsilon_{2}\right)^{3} \text {, } \\
& D_{1212 \varnothing \varnothing}\left(\tau, m, \epsilon_{1}, \epsilon_{2}\right)=\theta_{1}\left(\epsilon_{1}-m\right)^{4} \theta_{1}\left(-m-2 \epsilon_{2}\right)^{2} \\
& \theta_{1}\left(-m-\epsilon_{2}\right)^{4} \theta_{1}\left(-m+\epsilon_{1}-\epsilon_{2}\right)^{2} \text {, }
\end{aligned}
$$

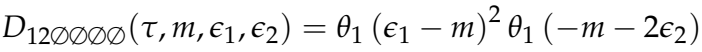

$$
\begin{aligned}
& \theta_{1}\left(-m-\epsilon_{2}\right)^{2} \theta_{1}\left(-m+\epsilon_{1}-\epsilon_{2}\right) \text {. }
\end{aligned}
$$




\section{CONFLICTS OF INTEREST}

The author declares no conflicts of interest.

\section{ACKNOWLEDGMENTS}

The author would like to thank Amer Iqbal for giving useful comments. The author is grateful to the referees for their very useful comments. Moreover, the support provided by the Abdus Salam School of Mathematical Sciences, Lahore, is gratefully acknowledged.

\section{References}

[1] B. Haghighat, A. Iqbal, C. Kozçaz, G. Lockhart, and C. Vafa, "M-Strings," Commun. Math. Phys. 334 no. 2, (2015) 779-842, arXiv: 1305.6322 [hep-th].

[2] B. Haghighat, C. Kozcaz, G. Lockhart, and C. Vafa, "Orbifolds of M-strings," Phys. Rev. D 89 no. 4, (2014) 046003, arXiv:1310.1185 [hep-th].

[3] S. Hohenegger and A. Iqbal, "M-strings, elliptic genera and $\mathcal{N}=4$ string amplitudes," Fortsch. Phys. 62 (2014) 155-206, arXiv: 1310.1325 [hep-th].

[4] S. Hohenegger, A. Iqbal, and S.-J. Rey, "M-strings, monopole strings, and modular forms," Phys. Rev. D 92 no. 6, (2015) 066005, arXiv:1503.06983 [hep-th].

[5] S. Hohenegger, A. Iqbal, and S.-J. Rey, "Instantonmonopole correspondence from M-branes on $\mathrm{S}^{1}$ and little string theory," Phys. Rev. D 93 no. 6, (2016) 066016, arXiv: 1511.02787 [hep-th].

[6] S. H. Katz and E. Sharpe, "D-branes, open string vertex operators, and Ext groups," Adv. Theor. Math. Phys. 6 (2003) 979-1030, arXiv: hep-th/0208104.

[7] J. Gomis, D. Rodriguez-Gomez, M. Van Raamsdonk, and H. Verlinde, "A Massive Study of M2-brane Proposals," JHEP 09 (2008) 113, arXiv : 0807.1074 [hep-th] .

[8] D. Gaiotto and E. Witten, "S-Duality of Boundary Conditions In N=4 Super Yang-Mills Theory," Adv. Theor. Math. Phys. 13 no. 3, (2009) 721-896, arXiv:0807.3720 [hep-th].

[9] R. Gopakumar and C. Vafa, "M theory and topological strings. 1.," arXiv:hep-th/9809187.

[10] R. Gopakumar and C. Vafa, "M theory and topological strings. 2.," arXiv: hep-th/9812127.

[11] T. J. Hollowood, A. Iqbal, and C. Vafa, "Matrix models, geometric engineering and elliptic genera," JHEP 03 (2008) 069, arXiv: hep-th/0310272.

[12] E. Sharpe, "Lectures on D-branes and sheaves," 7, 2003. arXiv:hep-th/0307245.

[13] S. Hohenegger, A. Iqbal, and S.-J. Rey, "Self-Duality and Self-Similarity of Little String Orbifolds," Phys. Rev. D 94 no. 4, (2016) 046006, arXiv: 1605.02591 [hep-th].

[14] M. Del Zotto, J. J. Heckman, A. Tomasiello, and C. Vafa, "6d Conformal Matter," JHEP 02 (2015) 054, arXiv:1407.6359 [hep-th].

[15] D. R. Morrison and W. Taylor, "Classifying bases for 6D Ftheory models," Central Eur. J. Phys. 10 (2012) 1072-1088, arXiv:1201.1943 [hep-th].

[16] T. Okazaki and D. J. Smith, "Topological M-strings and supergroup Wess-Zumino-Witten models," Phys. Rev. D 94 no. 6, (2016) 065016, arXiv:1512.06646 [hep-th].
[17] T. Okazaki and D. J. Smith, "Mock modular index of M2M5 brane systems," Phys. Rev. D 96 no. 2, (2017) 026017, arXiv:1612.07565 [hep-th].

[18] E. Witten, "Elliptic Genera and Quantum Field Theory," Commun. Math. Phys. 109 (1987) 525.

[19] E. Witten, "On the Landau-Ginzburg description of $\mathrm{N}=2$ minimal models," Int. J. Mod. Phys. A 9 (1994) 4783-4800, arXiv:hep-th/9304026.

[20] P. Berglund and M. Henningson, "Landau-Ginzburg orbifolds, mirror symmetry and the elliptic genus," Nucl. Phys. B 433 (1995) 311-332, arXiv: hep-th/9401029.

[21] M. Henningson, "N=2 gauged WZW models and the elliptic genus," Nucl. Phys. B 413 (1994) 73-83, arXiv: hep-th/9307040.

[22] D. Nemeschansky and N. P. Warner, "The Refined elliptic genus and Coulomb gas formulations of $\mathrm{N}=2$ superconformal coset models," Nucl. Phys. B 442 (1995) 623-654, arXiv:hep-th/9412187.

[23] K. Hosomichi and S. Lee, "Self-dual Strings and 2D SYM," JHEP 01 (2015) 076, arXiv: 1406.1802 [hep-th].

[24] A. Gadde and S. Gukov, "2d Index and Surface operators," JHEP 03 (2014) 080, arXiv: 1305.0266 [hep-th].

[25] A. Gadde, B. Haghighat, J. Kim, S. Kim, G. Lockhart, and C. Vafa, "6d String Chains," JHEP 02 (2018) 143, arXiv: 1504.04614 [hep-th].

[26] F. Benini, G. Bonelli, M. Poggi, and A. Tanzini, "Elliptic non-Abelian Donaldson-Thomas invariants of $\mathbb{C}^{3}$," arXiv: 1807.08482 [hep-th].

[27] H. Nakajima and K. Yoshioka, "Instanton counting on blowup. 1.," Invent. Math. 162 (2005) 313-355, arXiv:math/0306198. 